\title{
eNeuro
}

Research Article: Negative Results / Cognition and Behavior

\section{Oxytocin facilitates allo-parental behavior under stress in laboratory mice}

https://doi.org/10.1523/ENEURO.0405-21.2022

Cite as: eNeuro 2022; 10.1523/ENEURO.0405-21.2022

Received: 28 September 2021

Revised: 6 December 2021

Accepted: 4 January 2022

This Early Release article has been peer-reviewed and accepted, but has not been through the composition and copyediting processes. The final version may differ slightly in style or formatting and will contain links to any extended data.

Alerts: Sign up at www.eneuro.org/alerts to receive customized email alerts when the fully formatted version of this article is published.

Copyright @ 2022 Tsuneoka et al.

This is an open-access article distributed under the terms of the Creative Commons Attribution 4.0 International license, which permits unrestricted use, distribution and reproduction in any medium provided that the original work is properly attributed. 
Title Page

\section{Manuscript Title (50 word maximum)}

Oxytocin facilitates allo-parental behavior under stress in laboratory mice

\section{Abbreviated Title (50 character maximum)}

Oxytocin, vasopressin and parental care

\section{List all Author Names and Affiliations in order as they would appear in the published article} Yousuke Tsuneoka $^{12 \#}$, Chihiro Yoshihara ${ }^{1 \#}$, Ryuko Ohnishi ${ }^{13 \#}$, Sachine Yoshida ${ }^{12 \#}$, Eri Miyazawa ${ }^{1}$, Masanobu Yamada ${ }^{4}$, Kazuhiko Horiguchi ${ }^{4}$, W. Scott Young ${ }^{5}$, Katsuhiko Nishimori ${ }^{6}$, Tadafumi Kato ${ }^{7}$ and Kumi O. Kuroda ${ }^{1 *}$

${ }^{1}$ Laboratory for Affiliative Social Behavior, RIKEN Brain Science Institute, 351-0198 Japan

${ }^{2}$ Current address: Department of Anatomy, School of Medicine, Toho University, 143-8540, Japan

${ }^{3}$ Current address: Department of Bioscience and Biotechnology, Faculty of Agriculture, University of the Ryukyus, 903-0213, Japan

${ }^{4}$ Department of Internal Medicine, Division of Endocrinology and Metabolism, Gunma University Graduate School of Medicine, 3-39-15 Showa-machi, Maebashi, Gunma 371-8511, Japan.

${ }^{5}$ Section on Neural Gene Expression, National Institute of Mental Health, National Institutes of Health, Bethesda, MD, United States

7 Department of Obesity and Internal Inflammation, Fukushima Medical University, Fukushima 960-1295, Japan

${ }^{8}$ Department of Psychiatry and Behavioral Science, Graduate School of Medicine, Juntendo University, Tokyo, Japan.

\#: These authors contributed equally to this work.

\section{Author Contributions:}

YT, CY, RO, SY, KK designed and performed research, and analyzed data, with help from WSY, MY, KH, KN, TK. EM analyzed data. KK organized the study. WSY, MY, KH, KN, TK, YT, CY, RO, SY, EM, KK wrote the paper.

\section{Correspondence should be addressed to (include email address)}


$34{ }^{*}$ Contact: Kumi O. Kuroda, Laboratory for Affiliative Social Behavior, RIKEN Center for Brain 35 Science, 2-1 Hirosawa, Wako-shi, Saitama, 351-0198 Japan.

36 e-mail: kumi.kuroda@a.riken.jp

Tel: +81-48-467-6949, Fax: +81-48-467-6947

38

6. Number of Figures

7. Number of Tables

\section{Number of Multimedia}

0

9. Number of words for Abstract

\section{Number of words for Significance Statement}

\section{Number of words for Introduction}

\section{Number of words for Discussion}

\section{Acknowledgements}

This investigation was supported in part by RIKEN Brain Science Institute (2004-2017), RIKEN Center for Brain Science (2018-21 to KK), a Long-Term Fellowship of the Human Frontier Science

Program (2002-2004, KK), DRI Research Grant from RIKEN (2005-2006, KK), Research Facilitation Grant of Uehara Memorial Foundation, Japan (2008 to KK) and by JSPS KAKENHI Grant Numbers JP17790832, JP26282220, JP18H02710 to KK, JP21890307 to YT, and JP20890287

by the intramural research program of the NIMH 
67 (ZIAMH002498). We wish to thank Drs. Valery Grinevich, Geert de Vries, William Armstrong, and 68 Michael Numan for helpful discussion, Kashiko Tachikawa for initial breeding and phenotyping, 69 Sayaka Komatsu, Teppo Maruyama, Lana Ohkuma for technical assistance, and the RIKEN Center 70 for Brain Science, Research Resources Division for animal husbandry.

\section{Conflict of Interest}

The authors declare no competing financial interests.

\section{Funding sources}

This investigation was supported in part by RIKEN Brain Science Institute (2004-2017), RIKEN Center for Brain Science (2018-21 to KK), a Long-Term Fellowship of the Human Frontier Science Program (2002-2004, KK), DRI Research Grant from RIKEN (2005-2006, KK), Research Facilitation Grant of Uehara Memorial Foundation, Japan (2008 to KK) and by JSPS KAKENHI 80 Grant Numbers JP17790832, JP26282220, JP18H02710 to KK, JP21890307 to YT, and JP20890287 81 to SY. WSY research was supported by the intramural research program of the NIMH 82 (ZIAMH002498). 


\section{Abstract (max 250 words incl citations)}

Oxytocin (Oxt) controls reproductive physiology and various kinds of social behaviors, but the exact contribution of Oxt to different components of parental care still needs to be determined. Here we illustrate the neuroanatomical relations of the parental nurturing-induced neuronal activation with magnocellular oxytocin neurons and fibers in the medial preoptic area (MPOA), the brain region critical for parental and alloparental behaviors. We utilized genetically-targeted mouse lines for Oxt, oxytocin receptor (Oxtr), vasopressin receptor 1 a (Avprla), vasopressin receptor $1 b$ (Avprlb), and thyrotropin-releasing hormone (Trh) to systematically examine the role of Oxt-related signaling in pup-directed behaviors. The Oxtr-Avprla-Avprlb triple knockout (TKO), and Oxt-Trh-Avprla-Avprlb quadruple $\mathrm{KO}(\mathrm{QKO})$ mice were grossly healthy and fertile, except for their complete deficiency in milk ejection and modest deficiency in parturition secondary to maternal loss of the Oxt or Oxtr genes. In our minimal stress conditions, pup-directed behaviors in TKO and QKO mothers and fathers, virgin females and males were essentially indistinguishable from those of their littermates with other genotypes. However, Oxtr $\mathrm{KO}$ virgin females did show decreased pup retrieval in the pup-exposure assay performed right after restraint stress. This stress vulnerability in the Oxtr KO was abolished by the additional Avprlb KO. The general stress sensitivity, as measured by plasma cortisol elevation after restraint stress or by the behavioral performance in the open field and elevated plus maze, were not altered in the Oxtr KO but were reduced in the Avprlb KO females, indicating that the balance of neurohypophysial hormones affects the outcome of pup-directed behaviors.

\section{Significance Statement (max 120 words)}

Parental care without suckling induces the most significant transcriptional activation in the caregivers' anterior commissural nucleus, the third-largest population of oxytocin neurons in the medial preoptic area, but not in its non-oxytocinergic neurons. The pup-directed behaviors in postpartum mothers, fathers and virgin males and females of oxytocin receptor (Oxtr), vasopressin receptor (Avpr) $1 a$ and $1 b$ triple knockout mice were essentially normal in our standard experimental conditions with minimal stress. Under stressful conditions, however, Oxtr KO mice showed decreased parental nurturing behaviors, which was compensated for by combining with the Avprlb KO. 


\section{Introduction ( $\max 750$ words incl citations)}

Oxytocin (Oxt) and vasopressin (Avp) are nonapeptide hormones with a common ancestor gene. Avp is critically involved in osmoregulation and Oxt stimulates uterine contraction during parturition and milk-ejection during nursing (Robinson and Verbalis, 2003; Wakerley, 2005). In addition to these peripheral functions, Oxt and Avp have direct actions onto neurons expressing their receptors, and participate in the control of anxiety, pain and stress responses, social recognition, pair bonding, and aggression (Insel, 2010; Neumann and Landgraf, 2012; Onaka et al., 2012; Hurlemann and Grinevich, 2017; Grinevich and Neumann, 2020).

For maternal and allomaternal behaviors, there are numerous reports supporting the positive effects of oxytocin (Takayanagi et al., 2005; Caldwell and Young, 2006; Marlin et al., 2015). The facilitatory roles of Oxt in parental care are reported most frequently during the high-stress conditions, such as the onset/initial learning phase of parenting or in a non-home cage environment (for example, (Pedersen et al., 1982; Marlin et al., 2015; Carcea et al., 2021)). In these cases, however, Oxt facilitation of maternal behavior could be due to OT's anxiolytic / anti-stress effect in general, rather than via its primary role in maternal behavior per se (McCarthy et al., 1992; McCarthy, 1995; Yoshihara et al., 2017). Moreover, there are several studies reporting that multiple components of parental behavior are intact in mice harboring genetic mutations of the Oxt-Oxtr system (Nishimori et al., 1996; Young et al., 1996; Gross et al., 1998; Macbeth et al., 2010), impeding a coherent explanation of the exact role of oxytocin in different components of parental care. One of the confounding factors is the possible cross-activation/compensatory mechanisms of the Oxt and Avp systems via their receptors. Specifically, more than $80 \%$ structural homology between Oxtr and Avpr1a causes significant cross-activation (Manning et al., 2012; Jurek and Neumann, 2018), which is not negligible as both Oxt and Avp are implicated in maternal behavior (Bosch and Neumann, 2008).

From the viewpoint of functional neuroanatomy, we have previously studied the pattern of neuronal activation during maternal, paternal and allomaternal care in mice (Tsuneoka et al., 2013; Tsuneoka et al., 2015). We focused on the medial preoptic area (MPOA), the critical brain area for parental care (Numan, 1974; Numan and Numan, 1994; Numan, 2020), and investigated expression of c-Fos protein, the component of AP-1 transcription factor as a reliable readout of neuronal activation (Herdegen and Leah, 1998). The most pronounced c-Fos expression after two hours of nurturing behavior is observed at the anterior commissural nucleus (AC, previously abbreviated as $\mathrm{ACN}$ ) of the MPOA. The AC contains the third largest population of magnocellular Oxt neurons, Oxt fibers as well as Avp fibers, and thyrotropin-releasing hormone (Trh)-producing neurons (Peterson, 
149 1966; Armstrong et al., 1980; Rhodes et al., 1981; Sofroniew, 1985; Castel and Morris, 1988;

150 Grinevich and Akmayev, 1997). Both oxytocinergic and non-oxytocinergic AC neurons are c-Fos

151 positive after two hours of pup exposure in postpartum mothers. In virgin females, however, the

152 transcriptionally-activated AC neurons during allomaternal behavior are essentially

153 non-oxytocinergic, while roughly $40 \%$ of these activated AC neurons expressed calcitonin-receptor

154 (Calcr) (Yoshihara et al., 2021). Moreover, while Calcr neurons in the central part of the MPOA

155 (cMPOA), which lies ventrally to the AC, are functionally critical for parental nurturing (Yoshihara 156 et al., 2021), all non-oxytocinergic AC neurons including Calcr neurons are not. Still, the close 157 spatial correlation of the parenting-induced activation pattern and the distribution of oxytocin 158 neurons and fibers in the MPOA (see Fig. 1, 2 and (Tsuneoka et al., 2013)) is remarkable, and 159 prompted us to further investigate this issue. Here, to elucidate the exact role of Oxt system in 160 parental care, we used seven lines of genetic mutant mice in combination, covering genes for $O x t$, 161 Trh, Oxtr, and Avp receptors la (Avprla), and $1 b$ (Avprlb), and performed a systematic investigation 162 of the role of Oxt-Avp system in postpartum-maternal, -paternal and allomaternal behaviors, as well 163 as infanticide of virgin male mice (collectively called "pup-directed behaviors"). 


\section{Materials and Methods}

\section{Animals}

All animal experimentation was approved by and conducted in accordance with regulations of the Animal Experiment Judging Committee of RIKEN, which was based on the National Institutes of Health guide "Principles of Laboratory Animal Care" (NIH publication no. 85-23, revised 1985). The male C57BL/6J mice used for this study were obtained originally from the Jackson Laboratory. The oxyto cin (B6;129S-Oxt $t^{t m l W s y} / \mathrm{J} ;$ stock number 2713) (Young et al., 1996), Avprla (B6.129P2-Avprla ${ }^{\text {tmlDgen }} / \mathrm{J}$; stock number 5776) (Deltagen, 2005) and Avprlb KO mouse strains (B6;129X1-Avprlb ${ }^{t m l W s y} / \mathrm{J}$; stock number 6160) (Wersinger et al., 2002) were obtained from the Jackson Laboratory (Bar Harbor, ME). A Oxtr KO mouse strain described in (Takayanagi et al., 2005) and a Trh KO mouse strain described in (Yamada et al., 1997) were developed by co-authors and available from their laboratories. Another Avprla and $1 b$ double $\mathrm{KO}$ mouse strain

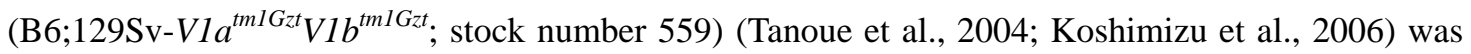
obtained from the Center for Animal Resources and Development, Kumamoto University (Kumamoto, Japan). All the mice, except for the Avprlb KO mouse in the quadruple KO (QKO) study, were backcrossed more than 7 times to C57BL/6J mouse. The Avprla (JAX 5776), Avpr1b (JAX 6160), Oxt and Trh quadruple KO mice line was named QKO; Avprla (JAX 5776), Avprlb (JAX 6160), and Oxtr triple KO mice line was named TKO; and Avprla, Avprlb (V1a ${ }^{t m l G z t} V 1 b^{t m l G z t}$, Kumamoto Univ. stock number 559), and Oxtr triple KO mice line was named HIR in this study.

Mice were raised in our breeding colony under controlled conditions (12 h light/dark cycle; lights on at 8:00 A.M.; $23 \pm 2{ }^{\circ} \mathrm{C} ; 55 \pm 10 \%$ humidity; and ad libitum access to water and food). Mice were weaned at 4 weeks of age and were housed in groups of four or five in ventilated shoebox cages $(267 \times 483 \times 152 \mathrm{~mm})$ with TEK-Fresh bedding (Harlan, Indianapolis, IN, USA). All mice were 1224 weeks old at the start of experiments.

For generating QKO mice, the Avprlb mouse line were a C57BL/6J $\times 129$ mixed background. For generating TKO mice, we used the Avprlb mouse line which were backcrossed 5 times to $\mathrm{C} 57 \mathrm{BL} / 6 \mathrm{~J}$ background. Breeding of genetic mutant mice were performed as described (Kuroda et al., 2011); briefly, first, double-KO mice of approximate combinations were produced by crossbreeding of single-KO mice. Then, we crossbred different combination of double-KO mice to produce QKO or TKO mice. The TKO male mice were crossbred to triple-heterozygous or single-KO/double-heterozygous female mice, and their littermate offspring were used for the behavioral testing. After the QKO male mice were obtained, they were crossbred to 
double-KO/double-heterozygous or single-KO/triple-heterozygous female mice, and the offspring were used for the behavioral testing.

The genotypes of mice were determined by electrophoresis of the product from polymerase chain reaction using Takara Ex Taq (Takara Bio Inc., Shiga, Japan) or Quick Taq ${ }^{\circledR}$ HS DyeMix (TOYOBO, Japan) with the specific primer sets for each mouse strain as described [Table 1]. The genomic DNA was obtained from last $2 \mathrm{~mm}$ of tail. Samples were put into $100 \mathrm{ul}$ of proteinase $\mathrm{K}$ solution (100mM Tris-HCl (pH 8.0), 5mM EDTA, 200mM NaCl, 0.2\% (w/v) SDS, $2 \%(\mathrm{v} / \mathrm{v})$ $15 \mathrm{mg} / \mathrm{ml}$ proteinase $\mathrm{K}$ (Takara, Japan)) and heated at $56{ }^{\circ} \mathrm{C}$ for 2 hours, $95^{\circ} \mathrm{C}$ for $2 \mathrm{~min}$, and then diluted by 300 ul distilled water. The genotyping were doubly confirmed after the end of each experiment.

\section{Assessment of pup-directed behaviors in standard condition}

For pup-exposure assays, two days before the first behavioral testing, mice were housed individually in clean cages with purified paper bedding (Alpha-Dri, Shepherd Specialty Papers, Watertown, TN, USA) and a piece of cotton square (Nestlet, Ancare, Bellmore, NY, USA) as nest material.

Pup-directed behaviors of mice were examined as described (Kuroda et al., 2011). Briefly, either three of their own pups or three unfamiliar pups 1-5 days old (donor pups) were gently introduced to the corner of the home cage of the subject mice avoiding the nest. Behavioral responses toward pups were observed as follows;

Latency to the first sniffing: the latency to when the subject sniffed a pup for the first time in a trial. Latency to the first pup retrieval: the latency to when the subject retrieved the first pup to the nest. Latency to the last pup retrieval: the latency to when the subject retrieved the last pup to the nest. If the subject retrieved all 3 pups to the nest, the retrieval of the $3^{\text {rd }}$ pup was considered as the last pup retrieval. If the subject retrieved 2 pups to where another pup was located and made a new nest there, the retrieval of the $2^{\text {nd }}$ pup was considered as the last pup retrieval. If the subject did not retrieve any pup, or retrieved only 1 or 2 pups and failed to retrieve the rest, the last pup retrieval time was regarded as a missing value.

Total time in nest for 3 pups: the sum of the duration of each pup being in the nest. If the subject retrieved 2 pups to where another pup was located and made a new nest there, the pup that was already in the new nest site was regarded as being in the nest since when the first pup was retrieved to the new nest site.

Pup grouping: collecting all three pups in the nest so that the pups contact each other. 
230 Full parental behavior: when the subject exhibited all of the following behaviors within a trial, it 231 was regarded as "full parental behavior": sniffing, retrieving and grouping all the pups, and 232 crouching over them in the nest for more than 1 minute.

Also, in the QKO, TKO, and HIR analyses, the subjects' behaviors were coded at $15 \mathrm{sec}$

234

235

236

237 intervals for pup sniffing, pup licking, nest building, retrieval, crouching over pups, and non-pup directed behaviors (such as feeding, resting, running in the cage). In addition, pups' audible distress calls induced by the subjects' contacts were noted. If the subject mice started to attack and bite the pups, all pups were immediately removed from the cage, and the subjects were deemed as "infanticidal". The attacked pups were immediately euthanized. For the QKO and TKO mothers (Fig. 4 and 5), the observation period was separated into two periods. The mother mice were observed for 15 min just after introduction of pups (first observation of the day); and then after a 15 min resting period with the introduced pups, they were again observed for $15 \mathrm{~min}$ (second observation of the day). The duration data from the first 15-min observation periods was used for analyses.

For the Oxt-Trh double KO (DKO) mice, four of the females used for the analyses of pup-directed behaviors were tested twice as a virgin, but only the results from the first trials were analyzed (Fig. 3A). These four females were not used for the further analyses. Other virgin females were tested only once as a virign. Of the DKO females used for the anlyses of postpartum pup-directed behaviors, only four were experienced the pup-exposure test as a virgin (whose results were included in Fig. 3A). The postpartum tests were performed either on the day of delivery or one day later. All the Oxt-Trh DKO male mice were tested once as virgins (Fig. 3I). Only males who exhibited infanticididal behavior as virgins were used for further analysis. Males were then cohabited with females. Some of these males were separated from their mates immediately after copulation (assessed by vaginal plugs), and tested on the day the paired females delivered (Fig. 3J). The other males were separated from their female mates after 7 days of cohabitation, and tested on the day the paired females delivered (Fig. 3L).

For the QKO and TKO female mice (Fig 4, 5), the responses to pups were first examined once per day for 4 straight days. Each female was subsequently paired with a sexually-experienced male C57BL/6J mouse to allow mating and delivery. The male mouse was changed to another in the case that the female did not become pregnant by 3 weeks cohabitation. On the day of delivery, the newborn pups were removed, and their location in the cage, general health, milk band and remaining fetal tissues were briefly examined according to the previous study (Kuroda et al., 2011). Then using their own 3 pups, the subject mothers were observed for their behavior as described above. In the case that the newborn pups were not cleaned properly, unhealthy or dead, donor pups were used 
instead. Use of donor pups does not sifnificantly affect pup exposure assay (Kuroda et al., 2011). If the mother showed any apparent abnormality of their movement caused by their parturition (observed in $O x t$ and Oxtr KO mice), we did not conduct the behavioral test on the day of delivery. On the next morning (PPD1), the resident pups in the home cage were gently removed and their conditions were checked again as they had been the day before (day of delivery). Then, the female mice were totally isolated from the pups. After the isolation, they were subjected to behavioral testing once again 13 days later.

For the TKO male mice and their littermates (Fig 7), the responses to pups were examined once before mating. Each male was subsequently paired with two parous C57BL/6J mice to allow mating. They were checked for vaginal plugs daily, and the cohabitation with females continued for 17 days after confirmation of the plugs to assure pregnancy as indicated by enlargement of abdomen. In the case that a female become pregnant without a plug, both females were removed from the mating cage. If there were no sign of pregnancy after a two-month cohabitation, the males were not used for the subsequent tests. One to two days after one of the paired females delivered, the male behavior was examined using their biological offspring once per day for 2 straight days, and once again 12-14 days later.

For the HIR female mice (Fig. 8A, B), different individuals were used for virgin female and mother mice assays. For virgin females, the responses to pups were first examined once per day for 3 straight days. For PPD0 mothers, they were subjected to behavioral testing on the day of delivery as described above, but with 3 donor pups instead of their own. After the testing, mother mice were totally isolated from the pups. Then they were subjected to behavioral testing for 2 straight days. For the HIR male mice (Fig 8C, D), different individuals were used for virgin male and father mice assays. For virgin males, the responses to pups were first examined once per day for 3 straight days. For father mice, they were cohabitated with female and own pups for 2 days after the delivery. Then, the father mice were totally isolated from the pups. They were subjected to behavioral testing once per day for 3 straight days, using 3 donor pups.

\section{Assessment of pup-directed behaviors after restraint stress}

Pup-directed behaviors immediately after acute restraint stress were first examined once per day for 4 straight days using virgin female TKO mice (Fig. 9). Restraint stress was given for 30 min using well-ventilated $50 \mathrm{ml}$ conical tubes. The mice were released into their own home cages after three unfamiliar pups of 1-5 days old were gently introduced to the corner of the cage of the subject mice avoiding the nest. Behavioral responses toward pups were observed in the same way as 
in the TKO virgin female assays under standard conditions as described in the previous section.

\section{Assessment of stress sensitivity}

Phenotypic behavioral differences between $\mathrm{Oxt}^{+/-}$and ${ }^{-/-}$, Avprla ${ }^{+/-}$and ${ }^{-/-}$, and Avprlb ${ }^{+/-}$ and ${ }^{-/}$mice were assessed with a specific behavioral and physiological test battery consisting of open-field (OF) and elevated plus maze (EPM) test using virgin male and female TKO mice for Figure 10. The cohort of mice were different from those in Figure 5-8. The OF and EPM tests were conducted basically as previously described (Kuroda et al., 2008) with minor modifications as described below. The effects of restraint stress on plasma corticosterone (CORT) levels were also examined.

\section{Open-field test}

The subjects were individually housed 2 days prior to the experiment, and were given white noise on the day before the experiment. An open-field monitoring system equipped with four monitoring channels was used (O'Hara \& Co., Ltd., Tokyo, Japan). Mice were placed in the center of the open field (50 cm x $50 \mathrm{~cm}, 40 \mathrm{~cm}$ high gray acrylic walls, bright-light condition of 70 lux) and allowed to explore for $15 \mathrm{~min}$. The distance traveled and precent of time at the center area of the field (size is $36 \%$ of the field) were measured using an automatic monitoring system Time OFCR4 (O’Hara \& Co., Ltd.).

2. Elevated plus maze test

A week after the OF test, the mice were tested in the EPM for $15 \mathrm{~min}$. The maze was set at a height of $50 \mathrm{~cm}$ above the floor and consisted of four arms $(25 \mathrm{~cm} 5 \mathrm{~cm})$, and a platform made of gray acrylic: two opposite arms were open, and the other two arms were enclosed by 15-cm-high transparent walls (room was illuminated at 70 lux). A mouse was placed in the center platform, positioned to face one of the open arms, and allowed to explore the maze for $15 \mathrm{~min}$. The time spent in the different arms and the numbers of arm entries were automatically analyzed using Time EP2 Two Maze System (O’Hara \& Co., Ltd.).

\section{Restraint stress and plasma CORT}

A week after the EPM assessments, half the subject mice were given acute restraint stress for 30 min using well-ventilated $50 \mathrm{ml}$ conical tubes. The rest of mice were left without stress for 30 min. Then the mice were decapitated and the trunk blood immediately collected was assayed for CORT by ELISA (Corticosterone, ELISA Kit, AssayMax; ASSAYPRO, MO, USA). Blood collection was performed in the morning, between 10:00-12:00. 


\section{Preparation of brain sections}

For histological analyses in Figure 6, TKO mice used for the Figure 5 experiments were deeply anesthetized with sodium pentobarbital $(50 \mathrm{mg} / \mathrm{kg}$, i.p.) and then perfused transcardially with 4\% (w/v) paraformaldehyde (PFA) in phosphate-buffered saline (PBS, pH 7.4). The brains were removed, immersed in the same fixative at $4^{\circ} \mathrm{C}$ overnight, followed by cryoprotection in the series of $20 \%$ and $30 \%$ (w/v) sucrose in PBS for two days, embedded in O.C.T. Compound (Sakura Finetek Japan, Tokyo, Japan), and stored at $-80^{\circ} \mathrm{C}$ until cryosectioning. Brains were cryosectioned coronally at a thickness of $40 \mu \mathrm{m}$ and evaluated using the mouse brain atlas (Franklin and Paxinos, 2007). Every third section from the serial sections was processed for immunohistochemistry (IHC).

\section{Immunohistochemistry}

IHC on free-floating sections was performed essentially as described (Tsuneoka et al., 2013). Single and double labelling was performed for immunohistochemical detection of neurophysin I (NPI), Avp, c-Fos and NeuN. To label Oxt neurons, anti-NPI was used, as Oxt and NPI are cleaved from the same precursor, preprooxyphysin. The anti-NPI antibody is more sensitive than an anti-Oxt antibody. The sections were washed with PBS containing 0.2\% Triton-100 (PBST), incubated with $0.3 \% \mathrm{H}_{2} \mathrm{O}_{2}$ in methanol for 5 minutes, washed with PBST, blocked with $0.8 \%$ Block Ace (Dainihon-Seiyaku, Osaka, Japan) in PBST, and incubated at $4{ }^{\circ} \mathrm{C}$ overnight with goat primary antibody against NPI (1:6000, sc-7810, Santa Cruz Biotechnology, Inc., Dallas, TX, USA). The following morning, the sections were washed and incubated with biotin-conjugated horse anti-goat secondary antibody (1:2000, BA-9500, Vector Laboratories, Inc., Burlingame, CA, USA) for 2 hours and then in $\mathrm{ABC}$ peroxidase reagent (Vectastain $\mathrm{ABC}$ Elite kit; Vector Laboratories) for 1 hour according to the manufacturer's instructions. The labelling was visualized by incubation in 3,3' diaminobenzidine (DAB) solution with nickel intensification (DAB peroxidase substrate kit, Vector Laboratories) for 5 minutes. For c-Fos-NPI and Avp-NeuN double staining, the first staining was processed similarly except the staining procedure used rabbit primary antibody against c-Fos (1:5000, sc-52, RRID:AB_2106783, Santa Cruz Biotechnology, Inc., Dallas, TX, USA) or Avp (1:5000, AB1565, Millipore, CA, USA), and biotin-conjugated horse anti-rabbit secondary antibody (1:2000, BA-1100, Vector Laboratories, Inc., Burlingame, CA, USA). The sections underwent the second staining, which used anti-NPI antibody, mouse anti-NeuN antibody (1:6000, MAB377, Millipore Corporation, Temecula, USA). For anti-NeuN double staining, horse anti-mouse secondary antibody (1:2000, BA-2000, Vector Laboratories, Inc., Burlingame, CA, USA), and the ABC alkaline phosphatase reagent (Vectastain ABC-AP kit, Vector Laboratories) were used. The brown signals 
were developed by 5 minutes of immersion in DAB solution without nickel, and pink color was developed by 5 minutes of immersion in Vector Red substrate (Vector red alkaline phosphatase substrate kit, Vector Laboratories). Subsequently, they were washed with PBS and then mounted on gelatin-coated slides using mounting medium (Vectashield; Vector Laboratories).

\section{Histological analysis}

For labeling of NPI-ir and c-Fos-ir cells, three rostral-to-caudal sections where the NPI and c-Fos expression were prominent were examined. Double- labeled sections of c-Fos and NPI were viewed under a brightfield microscope (Leica DM6000B; Leica Microsystems, Germany). The labeling protocol was described (Tsuneoka et al., 2013).

For analysis of NPI-ir and Avp-ir cells of TKO female mice (Fig. 6), brightfield photomicrographs were obtained using a digital slide scanner (NanoZoomer Digital Pathology; Hamamatsu Photonics, Shizuoka, Japan) with a 20-40x objective. The contrast and brightness of the all photographs were adjusted only linearly and uniformly for all the micrographs used in one experiment, using software (Image J (Rasband, 1997-2018)). Captured images were manually labelled by Image J. PVH and SO was identified by counterstaining using NeuN-ir. Anatomical nomenclature and classification were used according for reference (Watson et al., 2011).

\section{Statistical analysis}

All statistical analyses were conducted using R v. 3.6.3 (R Development Core Team, 2018). Welch's t-test and Fisher's exact test were used to compare continuous and categorical data, respectively. Multiple comparisons were conducted with P-values adjusted by Holm's method. Error bars are mean \pm standard error of mean unless otherwise specified. Datapoints of "censored", incomplete retrieval observations were replaced by the maximum observation time (30min for virgin females, virgin males, and fathers in Figure 4, 5, 7, 9, and mothers in Figure 8; 15 min for mothers in Figure 4, 5) (e.g., if a subject mouse retrieves only one pup within the session, the latencies for the second and third retrieval are regarded as $30 \mathrm{~min}$, rather than as missing datapoints. We also tried survival analyses, but comparing the effects of three genetic loci became complicated in the survival analysis. Please see also the Extended data Fig. 9-1 for another strategy, and the main conclusion is the same). The biological replicate number is the same as the number of samples, and the technical replicates are not applicable or taken in this study. All the statistical details and the numbers of animals used can be found either in the figure legends or results. 


\section{Results}

Neuroanatomical relations of the activated neurons during alloparenting of Oxt neurons and fibers in the preoptic and adjoining areas

Our initial motivation to study the relations between Oxt and parental behavior derived from the remarkable spatial correlation of neuronal activation after pup nurturing with the distribution of oxytocinergic neurons and fibers in the MPOA (Fig. 1, 2). We have already reported the quantification of each MPOA subregions for nurturing-induced activation (Tsuneoka et al., 2013), and here we illustrate anatomical and spatial relations between these neuronal activation with Oxt neurons and fibers. Observing c-Fos expression pattern, we found that parasagittal and horizontal sections consistently depicted the anatomical structures of the paraventricular nucleus of the hypothalamus (PVH) (arrowheads), AC (Box in Fig. 1A, 1B), and the c-Fos neuronal distributions after 2 hours (Fig1A, 1B) or 6 hours (Fig. 2) of pup nurturing (arrows) in C57BL/6 virgin female mice. c-Fos-ir neurons were found most densely in the AC, compared to the PVH, SO, and other MPOA subregions. Within the AC, Oxt-ir thick dendrites with a characteristic corkscrew-like morphology and irregular varicosities (Fig. 1B, arrows) were numerous (Castel and Morris, 1988). Even outside of the AC, the regions with dense c-Fos-ir neurons were colocalized with Oxt fibers, particularly in the preoptic area but not in the hypothalamus (for example, areas indicated by arrows in the ventral MPOA, Fig. 1, left, second; Fig. 2, left, first and second panels), indicating the spatial correlation between the non-Oxt MPOA neurons involved in nurturing behaviors and the Oxt neurons involved in parturition and lactation. In contrast, there were several spherical tissue masses (numbered as 1-4 in the Fig. 1A, 2) devoid of both c-Fos and Oxt fibers, suggesting that the developmental formation of the parenting-relevant anatomical structure was intermixed with other cell masses in the MPOA. Specifically, the tissue mass 1 roughly corresponds with the ventral part of the septohypothalamic area, mass 2 to the medial preoptic nucleus, mass 3 overlaps with the striohypothalamic area, and mass 4 overlaps with the posteroventral medial preoptic area and the ventral part of anterior hypothalamic nucleus. This unique organization might be formed by the embryonic preoptic-strial migration stream (Bayer and Altman, 2004).

In these virgin females, that were not suckled during brief pup nurturing, the induced c-Fos-ir were limited to non-oxytocinergic neurons. There were scarce c-Fos-ir Oxt neurons in the analyzed area that included the AC, PVH, PVPOA and SO (Fig. 1A, 1B, 2) of the pup-exposed virgin females (c-Fos-ir cells / Fos + Oxt double-ir cells $=0.067 \%$, Oxt-ir cells $/ \mathrm{c}-\mathrm{Fos}+$ Oxt double-ir cells $=0.915 \%$ ), in harmony with previous studies (Tsuneoka et al., 2013; Okabe et al., 2017). (Sheehan et al. found significant c-Fos induction in PVH by pup exposure (Sheehan et al., 2000), although they 
included the $\mathrm{AC}$ as the anterior magnocellular part of $\mathrm{PVH}$ ). It should be noted, however, that Fos expression itself does not imply the function of Oxt neurons. For example, while Oxt neurons are shown to be functional in facilitating social contacts among females, only about $1 \%$ of Oxt neurons (30\% of parvocellular Oxt neurons, which are about $\sim 3 \%$ of total Oxt neurons) in the PVH become c-Fos positive during social contacts (Tang et al., 2020).

\section{Delayed delivery of Oxt KO mice, and grossly-normal pup-directed behaviors of Trh-Oxt double mutant mice}

The above observations prompted us to directly address the role of Oxt and Avp systems in pup nurturing behaviors in mutant mice. Because normal maternal care was shown previously in three independent genetically targeted mouse lines of Oxt (Nishimori et al., 1996; Young et al., 1996; Gross et al., 1998), we hypothesized that there is functional redundancy of Oxt neurons in the AC. Neurons expressing Trh are distributed in the AC (Simerly et al., 1986), and the orthopaedia-expressing developmental progenitor cells are the same for Oxt and Trh neurons (Acampora et al., 1999). Moreover, Trh receptor (Trhr) was one of the transcripts upregulated in the dorsolateral MPOA of parenting mice in our previous DNA microarray study (Kuroda et al., 2007; Kuroda et al., 2008). These data prompted us to examine the maternal and alloparental behaviors of Oxt-Trh double KO (DKO) mice (Fig. 3).

Oxt-Trh DKO mice were born at a Mendelian ratio, and appeared healthy and normal as their littermates with other genotypes, with a minor retardation of body growth of Trh KO mice (Yamada et al., 1997). The majority of DKO virgin females also showed normal allomaternal behaviors (Fig. 3A). DKO female mice mated and became pregnant similarly to their non-KO (heterozygous or wildtype) littermates (Fig. 3B). However, a significant proportion (18 out of 35, $51.4 \%$ ) of $O x t \mathrm{KO}$ mothers showed severe delay of parturition, such that the labor continued for more than $24 \mathrm{hrs}$ after the delivery of the first pup, there was a dead pup found in the vaginal entry, or the maternal health deteriorated visibly, leading to spontaneous maternal death or euthanasia for the animal's welfare (5 out of 35, $14.3 \%$ ) (Fig. 3C). The delayed-labor phenotype of $O x t \mathrm{KO}$ mothers was not found in the initial studies in the mixed background (Nishimori et al., 1996; Young et al., 1996) but was suggested in a later study (Roizen et al., 2007), and significant in the present study in the C57BL/6 genetic background. These mothers that experienced abnormal labor were excluded from further analyses for maternal behaviors. The rate of abnormal labor was not significantly affected by the Trh genotype.

The home-cage postpartum maternal behaviors of $O x t \mathrm{KO}$ and DKO mothers, including 
nest building, placentophagia, and pup grouping were indistinguishable from those of their wild-type or heterozygous littermates (Fig. 3D-F). However, the pups born to Oxt KO mothers were weak, devoid of milk, and died within 2 days with $100 \%$ genetic penetrance (Fig. 3G; the 3 mothers with pups surviving through PPD1 lost them by PPD2), in concordance with previous literature (Nishimori et al., 1996; Young et al., 1996). At this time, we did not formally examine actual suckling behaviors; yet, the elongated nipples were found in all four $O x t \mathrm{KO}$ mothers examined, with no clear milk bands observed in their litters, suggesting that the nursing behaviors per se were performed by $\mathrm{Oxt}$ KO mothers. Among six $\mathrm{Oxt}^{+1}$ mothers examined, five mothers had elongated nipples, and four of them had the pups with a clear milk band. One $O x t$ heterozygous mother which did not have clearly elongated nipples had only one pup survive on the day of delivery.

Because unfed or unhealthy pups do not properly induce maternal retrieval, and because mouse mothers show maternal behaviors toward donor pups as well as their own pups, we performed pup retrieval assays with fed donor pups for all the subject mothers for consistency (see (Kuroda et al., 2011)). The Oxt KO mothers with or without Trh KO exhibited normal pup retrieval toward donor pups (Fig. $3 \mathrm{H})$.

We next examined pup-directed behaviors in Oxt-Trh DKO male mice. DKO virgin and post-mating (separated from females after copulation within a day) males performed infanticide as much as their littermates with other genotypes (Fig. 3I, J). After cohabitation with a female for 7 days, the proportion of males that successfully fertilized females was not significantly different among the genotypes (Fig. 3K). At the time of the females' deliveries, no differences in pup-directed behaviors were found between DKO and their littermates (Fig. 3L). As previously reported, C57BL/6 fathers need to stay with the pregnant mate female until late gestation to become mostly paternal (Tachikawa et al., 2013). Two Oxt KO males stayed with their pregnant mates until late gestation, as well as 3 males that stayed with their mates until delivery, turned paternal. Overall, these results indicate no abnormalities in pup-directed behaviors in DKO male and female mice.

\section{Pup-directed behaviors of Oxt-Trh-Avpr1a-Avpr1b quadruple KO (QKO) female mice}

Oxytocin's role in pup-directed behaviors might be obscured by interactions with the Avp system, possibly at the receptor level. Two kinds of Avp receptors, Avpr1a and Avpr1b, are expressed in the brain (Koshimizu et al., 2012). Therefore, we created Oxt-Trh-Avprla-Avprlb quadruple KO (QKO) mice, after crossing each line into the C57BL/6 genetic background at least four times.

During the breeding procedure, QKO male and female mice were born at a mendelian ratio, and appeared healthy. Moreover, they were grossly as fertile as their littermates with other genotypes, 
495

496

497

498

499

500

501

502

503

504

505

506

507

508

509

510

511

512

513

514

515

516

517

518

519

520

521

522

523

524

525

526

527

although we did not specifically examine their sexual behaviors in detail. The complete lack of milk transfer to the pups and the modest labor delay in Oxt KO mothers were consistently found throughout this study as shown in Fig. 3. To focus on maternal behaviors, we excluded from further analyses the mothers that experienced a severely disturbed labor.

The breeding procedure was inevitably complicated and laborious, because there are $3^{\wedge} 4$ (81) genotypes for the four gene loci. In this circumstance, testing all these genotypes separately was not practically feasible. Therefore, we categorized the genotypes into the following groups as in the Fig. 4: one comparison between QKO vs. all the other genotypes combined, and four comparisons for each gene locus as heterozygous vs. KO (e.g., QKO vs. Oxt ${ }^{-/} ; \operatorname{Trh}^{-/} ; \operatorname{Avprla}^{-/-} ; \operatorname{Avprlb}^{-/+}:$QKO vs. $O x t^{-/} ; \operatorname{Trh}^{-1-} ;$ Avprla ${ }^{-/+} ;$Avprlb $^{-/}$, etc. $)$.

Pup exposure assays were performed on four consecutive days in virgin females, on the day of delivery in the postpartum mothers and 13 days after the separation of pups at PPD1 (post-isolation, Fig. 4A). In essence, QKO females showed grossly indistinguishable nurturing behaviors, including latencies for first sniffing, first and last pup retrieval, and durations of crouching over pups, licking, and nest building (Fig. 4B-G). The consistent changes of pup-directed behaviors between genotypes in Fig. 4 and Fig. 5 were the increased licking behavior in Avprla KO (thus also QKO) mothers at PPD0. Anecdotally, we noted a mild tendency of polyurea by the soiled bedding of Avprla $\mathrm{KO}$ mice, even though normal basal urine output was reported for Avprla $\mathrm{KO}$ in a previous report in a genetically-mixed background (Koshimizu et al., 2006). The possible craving for water in Avprla $\mathrm{KO}$ and QKO postpartum mothers could have caused increased anogenital licking, and compensatory decrease of the crouching behavior. Other differences among genotypes were sporadic and not consistent (Fig. 5), suggesting the scarcity of robust deficits in maternal or allomaternal care of the QKO mutant females.

\section{Pup-directed behaviors of Oxtr-Avprla-Avprlb triple knockout (TKO) and}

\section{Oxt-Trh-Avpr1a-Avpr1b quadruple KO (QKO) female mice}

When the Oxtr KO mouse line (Takayanagi et al., 2005) became available to us, we created Oxtr-Avprla-Avprlb triple knockout (TKO) mice, after crossing each line into C57BL/6 genetic background at least four times, aiming at complete abolition of the Oxt-Avp system in the brain. First, we have examined if the lack of Oxtr or Avpr genes affected the expression of Oxt or Avp though compensatory mechanisms, as suggested previously (Vaidyanathan and Hammock, 2020). In our experimental conditions, no significant differences of the expression levels of Oxt and Avp between Oxtr KO and heterozygous females were detected in the AC, SO, or PVH (Fig. 6). 
As in $O x t \mathrm{KO}$ mothers, complete lack of milk transfer to the pups (Takayanagi et al., 2005) and the mild labor delay in Oxtr KO mothers were also observed throughout this study. Again, we examined allomaternal and postpartum maternal behaviors in TKO females, excluding the mothers with a severely disturbed labor.

Similar to the results from QKO, we did not find any robust defects in maternal and allomaternal care in TKO females. The only consistent statistical significance (Fig. 4) was found in the increased licking duration in Avprla KO and TKO PPD0 females. Nipple elongation, indicating suckling, was found in all nine Oxtr KO mothers examined on PPD0, and no clear milk band was observed in the pups' stomaches. In particular, pup-retrieval latencies, the reliably measurable index for parental motivation in mice (Kuroda et al., 2011), did not differ among genotypes for the first, second (data not shown) or the third pup (Fig. 4, 5) in either QKO, TKO or any single-locus KO females, indicating that there were no gross defects in pup retrieval in Oxt, Oxtr, Avprla, Avpr1b, Trh single KOs or in TKO or QKO females.

\section{Pup-directed behaviors in TKO male mice}

Next, we examined the pup-directed behaviors of male mice, focusing on TKO mutant lines. In virgin Oxtr KO males, a significant increase of parental behavior in virgin males and decrease of infanticide in fathers on PPD1 were observed (Fig. 7F). The frequency of infanticide is affected by various environmental stimuli and internal factors (Parmigiani and vom Saal, 1994), and it is possible that an anxiolytic effect of Oxt may cause this difference. Paternal behavior at two different timepoints, however, was not significantly affected by any of the single KOs or by the TKO (Fig. 7B-F), as seen in our female mice.

\section{Confirmation of the results in another Avprla and Avpr1b mutant strains}

The paucity of parental care-related phenotypes in the genetic mutants for the Oxt-Avp system so far identified was surprising. One concern about the genetic mutant line of Avprla used in the Figs. 3-7 was that it was not a null mutation, but an insertion mutation, and there was no obvious phenotype. This means that we have to rely solely on the PCR genotyping to segregate the mutant from the wild-type allele, unlike the Oxt and Oxtr mutants where lack of milk is $100 \%$ observable in mutant mothers. We therefore obtained another targeted-mutant line of Avprla which has been confirmed for the phenotype in the cardiovascular system (Egashira et al., 2004; Koshimizu et al., 2006), along with the Avprlb mutant line created in the same laboratory (Tanoue et al., 2004), and bred for the new TKO mice (i.e., the same mutant line for Oxtr, and independent lines for Avprla 
and $1 b$ from Fig. 5 and 7), termed HIR.

Examination of virgin female, postpartum, virgin male and paternal HIR TKO mice (Fig. 8) did not show any significant effects of any genotype on any pup-directed behaviors, except for the increased pup licking in one of three timepoints each in virgin and postpartum females, and decreased nest building behavior in one and two of the three timepoints each in virgin and postpartum females in the standard pup-exposure assay (Fig. 8A, B).

\section{Parental care of Oxtr KO is susceptible for physical stress}

So far, our experiments did not elucidate the significant role of Oxt-Avp system in parental care, even in combination with mutations of 8 related genetic loci, indicating that the genetic redundancy among these genes was unlikely the cause of unsuccessful detection of phenotypes in pup-directed behaviors. However, it was still possible that the role of Oxt-Avp system in parental nurturing was not readily detectable in the minimal-stress laboratory environment. Actually, parental nurturing is always in trade-off with other drives, such as hunger and self-security (Li et al., 2019; Yoshihara et al., 2021), but these competing needs do not occur in laboratory environments. Therefore, we next examined the parental care in more stressful conditions, and found a significant decrease of nurturing behaviors if the virgin females were tested right after $30 \mathrm{~min}$ of restraint stress (Fig. 9, Extended data Fig. 9-1). Three of the Oxtr KO virgin females exhibited infanticide toward donor pups after the restraint stress (Fig. 9A), although this effect did not reach statistical significance. The Oxtr KO virgin females also showed decreased pup retrieval, and thus the total nesting time summed for all the pups was reduced (Fig. 9D, E) in the pup-exposure assay performed right after restraint stress. On the other hand, this stress vulnerability caused by Oxtr KO was abolished by additional Avprlb KO (Fig. 9E). Rather opposite effects of Oxtr and Avpr1b were found on nurturing behavior under stress. Such distinct roles of Oxtr and Avpr1b has been demonstrated in fear control in the central amygdala (Huber et al., 2005) and with the Bruce effect (Wersinger et al., 2008), suggesting the importance of fine balance of neurohypophysial hormones on behavioral outcome in these complex contexts.

\section{Oxtr KO females exhibit normal stress reactivity in non-maternal context}

To determine whether the effect of Oxtr KO in disturbing alloparental care is secondary to the well-known general anxiolytic effect of Oxt or not, the general stress sensitivitie of the mutants were examined. Plasma cortisol levels after restraint stress, or the behavioral performances in the open field and elevated plus maze were not altered in Oxtr KO females (Fig. 10A-C). On the other 
594 hand, there was a significant increase of general anxiety in Avprlb KO females, as evidenced 595 consistently by the three measurements (Fig. 10A-C). This anxiogenic effect of Avprlb was limited 596 to females and not found in males, except for one measurement on the elevated plus maze (Fig. 597 10D-F). These results suggest that while the mild increase of alloparental nurturing after restraint 598 stress in Avprlb KO may be secondary to the general stress resistance, the decreased alloparenting 599 after stress in Oxtr $\mathrm{KO}$ is rather specific to a pup-nurturing context.

600

601 
602

603

604

605

606

607

608

609

610

611

612

613

614

615

616

617

618

619

620

621

622

623

624

625

626

627

628

629

630

631

632

633

634

\section{Discussion}

This study has shown that the genetic mutation in Oxtr causes deficits in alloparental nurturing specifically under a stressful condition. This finding is in harmony with the previous literature, which unequivocally demonstrated anti-stress and anxiolytic effects of Oxt in various conditions, including in a semi-natural environment (Ragnauth et al., 2005; Brunton and Russell, 2008; Neumann, 2008; Yoshida et al., 2009; Viviani et al., 2011; Knobloch et al., 2012; Sabihi et al., 2014; Menon et al., 2018). Environmental stress and risk factors should be inevitable in the feral life, thus this role of Oxt should be important for maintaining motivation to nurture. The differences between our findings with Oxtr KO mice and those of Takayanagi et al. (2005) may have been due to the different stress levels involved in pup exposure assays (see (Yoshihara et al., 2017) for details).

We also observed the modest deficits in parturition by either the Oxt or Oxtr mutation, a phenotype that was not observed in the original KO studies (Nishimori et al., 1996; Young et al., 1996; Gross et al., 1998; Takayanagi et al., 2005). However, this phenotype was observed partly in later studies (Roizen et al., 2007; Yoshida et al., 2019), possibly because of the difference in genetic backgrounds used. On the other hand, the complete lack of milk ejection despite vigorous suckling by starving pups was confirmed as originally reported (Nishimori et al., 1996; Young et al., 1996; Gross et al., 1998; Takayanagi et al., 2005).

Except for these phenotypes, however, this study has revealed the relative paucity of effects of $O x t-A v p$ genetic targeting on pup-directed behaviors in laboratory mice. In particular, we have found almost no robust facilitatory role of Oxt on maternal or allomaternal nurturing behaviors under our standard laboratory condition. And the TKO and QKO phenotypes indicate that the normal parental care is not due to the compensation for the congenital lack of Oxt or Oxtr by Avp or Trh molecular signaling. Of course, the compensation may occur via non-Avpr1a, non-Avpr1b, non-Trh mechanism. In contrast to the inability to compensate for the deficit in milk ejection due to the lack of $O x t$ or Oxtr, these data provide no evidence for Oxt-Avp-Trh's role in parental care, at least after chronic loss. Whether acute loss of one, two or all three neuropeptide signalling systems would affect parental care can be tested by acute molecular knockdown through AAV-mediated RNA interference, for example.

This study has several limitations other than the use of unnaturally, relatively stress-free laboratory conditions. For example, unlike laboratory mouse strains, virgin female wild mice are often not spontaneously maternal, and even unresponsive or infanticidal toward unfamiliar pups (McCarthy and vom Saal, 1985; Soroker and Terkel, 1988; Chalfin et al., 2014). Moreover, domesticated mice show differences in Oxt and Avp expression in brain regions known to regulate 
635

636

637

638

639

640

641

642

643

644

645

646

647

648

649

650

651

652

653

654

655

656

657

658

659

660

661

662

663

664

665

666

667

social behavior and emotion (Ruan and Zhang, 2016). Therefore, inbreeding and domestication may have rendered lab mice even less dependent upon the facilitatory effects of Oxt for the initiation of maternal behavior in inexperienced (first-time) mothers under standard laboratory conditions.

Second, the studied functions of Oxt neurons may be mediated by co-expressing neurotransmitters or neuropeptides, such as glutamate (Xu et al., 2020), corticotropin-releasing factor, cholecystokinin and dynorphin (Levin and Sawchenko, 1993). If this is the case, while Oxt molecules are not important, Oxt neurons may be important, and the function of Oxt neurons on pup-directed behaviors should be detected by manipulation of Oxt expressing neuronal activity by using AAV-mediated tetanus toxin or Gi-DREADD, for example, as performed in previous work (Tang et al., 2020).

Lastly, Oxt function may be more visible in nursing behavior and/or in the later lactation period. We observed nipple elongation, evidence for nursing on the day of delivery, consistent with the previous finding with a forebrain-specific Oxtr KO (Macbeth et al., 2010). However we did not formally exclude other possible problems in nursing-related behaviors. In particular, the Oxt neurons could be involved in the phenomenon called "Pavlovian milk conditioning". It has been known anecdotally that experienced lactating human and dairy animal mothers let down milk with conditional stimuli, such as a baby cry or smell, and bells signaling milking time in a dairy farm, all prior to physical suckling (Grosvenor and Mena, 1972; McNeilly et al., 1983; Fuchs et al., 1987; Tancin et al., 2001; Domjan, 2005). Once this conditioning is formed, the mothers may have milk letdown just by approaching and receiving infant sensory cues, or in human cases, just to think about the infants, prior to actual suckling by the infants, thus facilitating milk transfer to the infants. We recently identified a key player in maternal care, calcitonin receptor (Calcr)-expressing neurons in the cMPOA and the AC (Yoshihara et al., 2021). Calcr-expressing neurons are significantly activated during and required for maternal and allomaternal care. The close spatial relationship of Oxt neurons and Calcr neurons in the AC suggests that simultaneous firing of these neurons should occur repeatedly in the AC during maternal care, and may form the synaptic connections between these neuronal populations, which may be responsible for the Pavlovian milk conditioning. If this is the case, it may best explain why there is a striking co-distribution of the abundant activated non-oxytocinergic neurons among Oxt neurons in the AC and Oxt-fibers in the MPOA.

Even positive reports for Oxt's facilitation of parental care show relatively mild effects on specific components of parental care (Pedersen et al., 1994; Takayanagi et al., 2005; Marlin et al., 2015; Carcea et al., 2021), rather than abolishing many aspects of complex parental behaviors. Further investigations are needed to examine these possibilities and determine the exact role of Oxt 
668 system in pup-directed behaviors. However, at least the present findings provide a caution for the 669 pervasive, yet not-well substantiated, claim of an indispensable role of Oxt in all kinds of parental 670 care.

671 
672

673

674

675

676

677

678

679

680

681

682

683

684

685

686

687

688

689

690

691

692

693

694

695

696

697

698

699

700

701

702

703

704

705

706

707

708

709

\section{References}

Acampora D, Postiglione MP, Avantaggiato V, Di Bonito M, Vaccarino FM, Michaud J, Simeone A (1999) Progressive impairment of developing neuroendocrine cell lineages in the hypothalamus of mice lacking the Orthopedia gene. Genes Dev 13:2787-2800.

Armstrong WE, Warach S, Hatton GI, McNeill TH (1980) Subnuclei in the rat hypothalamic paraventricular nucleus: a cytoarchitectural, horseradish peroxidase and immunocytochemical analysis. Neuroscience 5:1931-1958.

Bayer SA, Altman J (2004) Development of the telencephalon: neural stem cells, neurogenesis, and neuronal migration. In: The rat nervous system, 3 Edition (Paxinos G, ed), pp 27-73. San Diego: Elsevier.

Bosch OJ, Neumann ID (2008) Brain vasopressin is an important regulator of maternal behavior independent of dams' trait anxiety. Proc Natl Acad Sci U S A 105:17139-17144.

Brunton PJ, Russell JA (2008) Keeping oxytocin neurons under control during stress in pregnancy. Prog Brain Res 170:365-377.

Caldwell HK, Young WS (2006) Oxytocin and Vasopressin: Genetics and Behavioral Implications. In: Handbook of neurochemistry and molecular neurobiology (Abel L, Lim R, eds), pp 573-607. Berlin: Springer-Verlag.

Carcea I et al. (2021) Oxytocin neurons enable social transmission of maternal behaviour. Nature.

Castel M, Morris JF (1988) The neurophysin-containing innervation of the forebrain of the mouse. Neuroscience 24:937-966.

Chalfin L, Dayan M, Levy DR, Austad SN, Miller RA, Iraqi FA, Dulac C, Kimchi T (2014) Mapping ecologically relevant social behaviours by gene knockout in wild mice. Nat Commun 5:4569.

Deltagen I (2005) NIH initiative supporting placement of Deltagen, Inc. mice into public repositories. MGI Direct Data Submission. In.

Domjan M (2005) Pavlovian conditioning: a functional perspective. Annu Rev Psychol 56:179-206.

Egashira N, Tanoue A, Higashihara F, Mishima K, Fukue Y, Takano Y, Tsujimoto G, Iwasaki K, Fujiwara M (2004) V1a receptor knockout mice exhibit impairment of spatial memory in an eight-arm radial maze. Neurosci Lett 356:195-198.

Franklin KBJ, Paxinos G (2007) The mouse brain in stereotaxic coordinates, 3rd Edition. San Diego: Academic Press.

Fuchs AR, Ayromlooi J, Rasmussen AB (1987) Oxytocin response to conditioned and nonconditioned stimuli in lactating ewes. Biol Reprod 37:301-305.

Grinevich V, Akmayev I (1997) An accessory magnocellular nucleus, anterior commissural nucleus, in the rat hypothalamus: immunohistochemical, tract-tracing, in situ hybridization, and experimental studies. Biogenic Amines 13:333-348.

Grinevich V, Neumann ID (2020) Brain oxytocin: how puzzle stones from animal studies translate into psychiatry. Mol Psychiatr. 
Gross GA, Imamura T, Luedke C, Vogt SK, Olson LM, Nelson DM, Sadovsky Y, Muglia LJ (1998) Opposing actions of prostaglandins and oxytocin determine the onset of murine labor. Proc Natl Acad Sci U S A 95:11875-11879.

Grosvenor CE, Mena F (1972) Evidence that the litter exerts an inhibitory influence on milk secretion in the rat during late lactatio. Fed Proc (Fed Am Stud Exp Biol) 31:275.

Herdegen T, Leah JD (1998) Inducible and constitutive transcription factors in the mammalian nervous system: control of gene expression by Jun, Fos and Krox, and CREB/ATF proteins. Brain Res Brain Res Rev 28:370-490.

Huber D, Veinante P, Stoop R (2005) Vasopressin and oxytocin excite distinct neuronal populations in the central amygdala. Science 308:245-248.

Hurlemann R, Grinevich V (2017) Behavioral pharmacology of neuropeptides : oxytocin, 1st edition. Edition. New York, NY: Springer Berlin Heidelberg.

Insel TR (2010) The challenge of translation in social neuroscience: a review of oxytocin, vasopressin, and affiliative behavior. Neuron 65:768-779.

Jurek B, Neumann ID (2018) The Oxytocin Receptor: From Intracellular Signaling to Behavior. Physiol Rev 98:1805-1908.

Knobloch HS, Charlet A, Hoffmann LC, Eliava M, Khrulev S, Cetin AH, Osten P, Schwarz MK, Seeburg PH, Stoop R, Grinevich V (2012) Evoked axonal oxytocin release in the central amygdala attenuates fear response. Neuron 73:553-566.

Koshimizu TA, Nakamura K, Egashira N, Hiroyama M, Nonoguchi H, Tanoue A (2012) Vasopressin V1a and V1b receptors: from molecules to physiological systems. Physiol Rev 92:1813-1864.

Koshimizu Ta, Nasa Y, Tanoue A, Oikawa R, Kawahara Y, Kiyono Y, Adachi T, Tanaka T, Kuwaki T, Mori T, Takeo S, Okamura H, Tsujimoto G (2006) V1a vasopressin receptors maintain normal blood pressure by regulating circulating blood volume and baroreflex sensitivity. Proceedings of the National Academy of Sciences 103:7807-7812.

Kuroda KO, Meaney MJ, Uetani N, Kato T (2008) Neurobehavioral basis of the impaired nurturing in mice lacking the immediate early gene FosB. Brain Res 1211:57-71.

Kuroda KO, Tachikawa K, Yoshida S, Tsuneoka Y, Numan M (2011) Neuromolecular basis of parental behavior in laboratory mice and rats: with special emphasis on technical issues of using mouse genetics. Prog Neuropsychopharmacol Biol Psychiatry 35:1205-1231.

Kuroda KO, Meaney MJ, Uetani N, Fortin Y, Ponton A, Kato T (2007) ERK-FosB signaling in dorsal MPOA neurons plays a major role in the initiation of parental behavior in mice. Mol Cel Neurosci $36: 121-131$.

Levin MC, Sawchenko PE (1993) Neuropeptide co-expression in the magnocellular neurosecretory system of the female rat: Evidence for differential modulation by estrogen. Neuroscience 54:1001-1018.

Li XY, Han Y, Zhang W, Wang SR, Wei YC, Li SS, Lin JK, Yan JJ, Chen AX, Zhang X, Zhao ZD, Shen WL, Xu XH (2019) AGRP Neurons Project to the Medial Preoptic Area and Modulate Maternal Nest-Building. J Neurosci 39:456-471. 
Macbeth AH, Stepp JE, Lee HJ, Young WS, 3rd, Caldwell HK (2010) Normal maternal behavior, but increased pup mortality, in conditional oxytocin receptor knockout females. Behav Neurosci 124:677-685.

Manning M, Misicka A, Olma A, Bankowski K, Stoev S, Chini B, Durroux T, Mouillac B, Corbani M, Guillon G (2012) Oxytocin and vasopressin agonists and antagonists as research tools and potential therapeutics. J Neuroendocrinol 24:609-628.

Marlin BJ, Mitre M, D'Amour J A, Chao MV, Froemke RC (2015) Oxytocin enables maternal behaviour by balancing cortical inhibition. Nature 520:499-504.

McCarthy MM (1995) Estrogen modulation of oxytocin and its relation to behavior. Adv Exp Med Biol 395:235-245.

McCarthy MM, vom Saal FS (1985) The influence of reproductive state on infanticide by wild female house mice (Mus musculus). Physiol Behav 35:843-849.

McCarthy MM, Kow LM, Pfaff DW (1992) Speculations concerning the physiological significance of central oxytocin in maternal behavior. Ann N Y Acad Sci 652:70-82.

McNeilly AS, Robinson IC, Houston MJ, Howie PW (1983) Release of oxytocin and prolactin in response to suckling. Br Med J (Clin Res Ed) 286:257-259.

Menon R, Grund T, Zoicas I, Althammer F, Fiedler D, Biermeier V, Bosch OJ, Hiraoka Y, Nishimori K, Eliava M, Grinevich V, Neumann ID (2018) Oxytocin Signaling in the Lateral Septum Prevents Social Fear during Lactation. Curr Biol 28:1066-1078 e1066.

Neumann ID (2008) Brain oxytocin: a key regulator of emotional and social behaviours in both females and males. J Neuroendocrinol 20:858-865.

Neumann ID, Landgraf R (2012) Balance of brain oxytocin and vasopressin: implications for anxiety, depression, and social behaviors. Trends Neurosci 35:649-659.

Nishimori K, Young LJ, Guo Q, Wang Z, Insel TR, Matzuk MM (1996) Oxytocin is required for nursing but is not essential for parturition or reproductive behavior. Proc Natl Acad Sci U S A 93:11699-11704.

Numan M (1974) Medial preoptic area and maternal behavior in the female rat. J Comp Physiol Psychol 87:746-759.

Numan M (2020) The Parental Brain: Mechanisms, Development, and Evolution: Oxford University Press.

Numan M, Numan MJ (1994) Expression of Fos-like immunoreactivity in the preoptic area of maternally behaving virgin and postpartum rats. Behav Neurosci 108:379-394.

Okabe S, Tsuneoka Y, Takahashi A, Ooyama R, Watarai A, Maeda S, Honda Y, Nagasawa M, Mogi K, Nishimori K, Kuroda M, Koide T, Kikusui T (2017) Pup exposure facilitates retrieving behavior via the oxytocin neural system in female mice. Psychoneuroendocrinology 79:20-30.

Onaka T, Takayanagi Y, Yoshida M (2012) Roles of oxytocin neurones in the control of stress, energy metabolism, and social behaviour. J Neuroendocrinol 24:587-598.

Parmigiani S, vom Saal FS, eds (1994) Infanticide and parental care: Harwood academic publishers.

Pedersen CA, Ascher JA, Monroe YL, Prange AJ (1982) Oxytocin Induces Maternal-Behavior in Virgin 
Female Rats. Science 216:648-650.

Pedersen CA, Caldwell JD, Walker C, Ayers G, Mason GA (1994) Oxytocin activates the postpartum onset of rat maternal behavior in the ventral tegmental and medial preoptic areas. Behav Neurosci 108:1163-1171.

Peterson RP (1966) Magnocellular neurosecretory centers in the rat hypothalamus. J Comp Neurol 128:181-190.

Pohlert, T. (2014). The Pairwise Multiple Comparison of Mean Ranks Package (PMCMR). https://CRAN.R-project.org/package=PMCMR

R Development Core Team (2018) R: A Language and Environment for Statistical Computing. Vienna, Austria: R Foundation for Statistical Computing.

Ragnauth AK, Devidze N, Moy V, Finley K, Goodwillie A, Kow LM, Muglia LJ, Pfaff DW (2005) Female oxytocin gene-knockout mice, in a semi-natural environment, display exaggerated aggressive behavior. Genes Brain Behav 4:229-239.

Rasband WS (1997-2018) ImageJ. In: U. S. National Institutes of Health, Bethesda, Maryland, USA.

Rhodes CH, Morrell JI, Pfaff DW (1981) Immunohistochemical analysis of magnocellular elements in rat hypothalamus: distribution and numbers of cells containing neurophysin, oxytocin, and vasopressin. J Comp Neurol 198:45-64.

Robinson AG, Verbalis JG (2003) Posterior pituitary gland. In: Williams textbook of endocrinology, 10th Edition (Williams RH, Larsen PR, eds), pp 281-329. Philadelphia, Pa.: Saunders.

Roizen J, Luedke CE, Herzog ED, Muglia LJ (2007) Oxytocin in the Circadian Timing of Birth. PLoS ONE 2.

Ruan C, Zhang Z (2016) Laboratory domestication changed the expression patterns of oxytocin and vasopressin in brains of rats and mice. Anatomical Science International 91:358-370.

Sabihi S, Durosko NE, Dong SM, Leuner B (2014) Oxytocin in the prelimbic medial prefrontal cortex reduces anxiety-like behavior in female and male rats. Psychoneuroendocrinology 45:31-42.

Sheehan TP, Cirrito J, Numan MJ, Numan M (2000) Using c-Fos immunocytochemistry to identify forebrain regions that may inhibit maternal behavior in rats. Behav Neurosci 114:337-352.

Simerly RB, Gorski RA, Swanson LW (1986) Neurotransmitter specificity of cells and fibers in the medial preoptic nucleus: an immunohistochemical study in the rat. J Comp Neurol 246:343-363.

Sofroniew MV (1985) Vasopressin- and neurophysin-immunoreactive neurons in the septal region, medial amygdala and locus coeruleus in colchicine-treated rats. Neuroscience 15:347-358.

Soroker V, Terkel J (1988) Changes in incidence of infanticidal and parental responses during the reproductive cycle in male and female wild mice Mus musculus. Anim Behav 36:1275-1281.

Tachikawa KS, Yoshihara Y, Kuroda KO (2013) Behavioral transition from attack to parenting in male mice: a crucial role of the vomeronasal system. J Neurosci 33:5120-5126.

Takayanagi Y, Yoshida M, Bielsky IF, Ross HE, Kawamata M, Onaka T, Yanagisawa T, Kimura T, Matzuk MM, Young LJ, Nishimori K (2005) Pervasive social deficits, but normal parturition, in oxytocin receptor-deficient mice. Proc Natl Acad Sci U S A 102:16096-16101. 
Tancin V, Kraetzl W-D, Schams D, Bruckmaier RM (2001) The effects of conditioning to suckling, milking and of calf presence on the release of oxytocin in dairy cows. Applied Animal Behaviour Science 72:235-246.

Tang Y et al. (2020) Social touch promotes interfemale communication via activation of parvocellular oxytocin neurons. Nat Neurosci 23:1125-1137.

Tanoue A, Ito S, Honda K, Oshikawa S, Kitagawa Y, Koshimizu TA, Mori T, Tsujimoto G (2004) The vasopressin V1b receptor critically regulates hypothalamic-pituitary-adrenal axis activity under both stress and resting conditions. J Clin Invest 113:302-309.

Tsuneoka Y, Maruyama T, Yoshida S, Nishimori K, Kato T, Numan M, Kuroda KO (2013) Functional, anatomical, and neurochemical differentiation of medial preoptic area subregions in relation to maternal behavior in the mouse. J Comp Neurol 521:1633-1663.

Tsuneoka Y, Tokita K, Yoshihara C, Amano T, Esposito G, Huang AJ, Yu LM, Odaka Y, Shinozuka K, McHugh TJ, Kuroda KO (2015) Distinct preoptic-BST nuclei dissociate paternal and infanticidal behavior in mice. EMBO J 34:2652-2670.

Vaidyanathan R, Hammock EAD (2020) Oxytocin receptor gene loss influences expression of the oxytocin gene in C57BL/6J mice in a sex- and age-dependent manner. J Neuroendocrinol 32:e12821.

Viviani D, Charlet A, van den Burg E, Robinet C, Hurni N, Abatis M, Magara F, Stoop R (2011) Oxytocin selectively gates fear responses through distinct outputs from the central amygdala. Science 333:104-107.

Wakerley JB (2005) Milk ejection and its control. In: Knobil and Neill's Physiology of reproduction, 3 Edition (Neill JD, ed), pp 3129-3190: Elsevier.

Watson C, Paxinos G, Puelles E (2011) The Mouse Nervous System: Academic press.

Wersinger SR, Temple JL, Caldwell HK, Young WS, 3rd (2008) Inactivation of the oxytocin and the vasopressin (Avp) 1b receptor genes, but not the Avp 1a receptor gene, differentially impairs the Bruce effect in laboratory mice (Mus musculus). Endocrinology 149:116-121.

Wersinger SR, Ginns EI, O'Carroll AM, Lolait SJ, Young WS, 3rd (2002) Vasopressin V1b receptor knockout reduces aggressive behavior in male mice. Mol Psychiatry 7:975-984.

Wickham, H. (2016). ggplot2. Elegant Graphics for Data Analysis. Springer-Verlag New York.

Xu S, Yang H, Menon V, Lemire AL, Wang L, Henry FE, Turaga SC, Sternson SM (2020) Behavioral state coding by molecularly defined paraventricular hypothalamic cell type ensembles. Science 370 .

Yamada M, Saga Y, Shibusawa N, Hirato J, Murakami M, Iwasaki T, Hashimoto K, Satoh T, Wakabayashi K, Taketo MM, Mori M (1997) Tertiary hypothyroidism and hyperglycemia in mice with targeted disruption of the thyrotropin-releasing hormone gene. Proc Natl Acad Sci U S A 94:10862-10867.

Yoshida M, Takayanagi Y, Inoue K, Kimura T, Young LJ, Onaka T, Nishimori K (2009) Evidence that oxytocin exerts anxiolytic effects via oxytocin receptor expressed in serotonergic neurons in mice. J Neurosci 29:2259-2271.

Yoshida M, Takayanagi Y, Ichino-Yamashita A, Sato K, Sugimoto Y, Kimura T, Nishimori K (2019) Functional Hierarchy of Uterotonics Required for Successful Parturition in Mice. Endocrinology 
862

863

864

865

866

867

868

869

870

871

872

873

874

160:2800-2810.

Yoshihara C, Numan M, Kuroda KO (2017) Oxytocin and Parental Behaviors. In: Behavioral pharmacology of neuropeptides : oxytocin (Hurlemann R, Grinevich V, eds), pp 119-153: Curr Top Behav Neurosci.

Yoshihara C, Tokita K, Maruyama T, Kaneko M, Tsuneoka Y, Fukumitsu K, Miyazawa E, Shinozuka K, Huang AJ, Nishimori K, McHugh TJ, Tanaka M, Itohara S, Touhara K, Miyamichi K, Kuroda KO (2021) Calcitonin receptor signaling in the medial preoptic area enables risk-taking maternal care. Cell Rep 35:109204.

Young WS, 3rd, Shepard E, Amico J, Hennighausen L, Wagner KU, LaMarca ME, McKinney C, Ginns EI (1996) Deficiency in mouse oxytocin prevents milk ejection, but not fertility or parturition. J Neuroendocrinol 8:847-853. 
875

876

877

878

879

880

881

882

883

884

885

886

887

888

889

890

891

892

893

894

895

896

897

898

899

900

901

902

903

904

905

906

907

\section{Figure/Table/Extended Data Legends}

Figure 1. Sagittal view of anatomical distribution of oxytocin neurons, fibers and parenting-induced c-Fos expression

Distribution of neurophysin I (NPI) (brown)-ir and c-Fos (black)-ir cell in and around of MPOA of virgin females after pup exposure (parasagittal section).

A: Left and right panels show representative photographs and their diagrammatic drawings, respectively. These sections were stained by IHC. Black squares and plus (+) symbols respectively represent strongly and weakly expressed c-Fos-ir neurons without NPI-ir. Filled and open red circles respectively represent strongly and weakly NPI-ir cell bodies without c-Fos signals. Green squares respectively represent NPI-ir cell bodies with c-Fos-ir. Red lines represent NPI-ir fibers. Numbers 1-4 show the areas relatively devoid of c-Fos-ir, NPI-ir neurons and fibers. Arrows indicate Oxt-ir thick dendrites with a corkscrew-like morphology and irregular varicosities. Panels are arranged in the medial-lateral order, from the left top to left bottom, and the right top to right bottom.

B: The high-magnification image of the blue squared region in (A), containing the AC. The circle-headed arrow indicate double-labeled cells of NPI and c-Fos. White arrowheads indicate single-labeled cells of c-Fos, black arrowheads indicate single-labeled cells of NPI, and small arrows indicate NPI-ir fibers.

Figure 2. Horizontal view of anatomical distribution of oxytocin neurons, fibers and parenting-induced c-Fos expression

Distribution of NPI (brown)-ir and c-Fos (black)-ir cell in and around of MPOA of virgin females after pup exposure (horizontal section). Left and right panels show representative photographs and their diagrammatic drawings, respectively. These sections were stained by IHC. Black squares and plus symbols respectively represent strongly and weakly expressed c-Fos-ir neurons. Filled and open red circles respectively represent NPI-ir cell bodies with or without c-Fos signals. Red lines represent NPI-ir fibers. Numbers 1-4 show the areas relatively devoid of c-Fos-ir, NPI-ir neurons and fibers. All panels are arranged in Ventral-Dorsal order from the left, top to bottom, and then the right, top to bottom.

Figure 3. Parental behavior, fertility, and abnormal delivery of the Trh-Oxt double KO (DKO) 
908

909

910

911

912

913

914

915

916

917

918

919

920

921

922

923

924

925

926

927

928

929

930

931

932

933

934

935

936

937

938

939

940

mice.

A: Responses to pup exposure in virgin females.

B: Fertility ratio of the females after mating.

C: Proportion of abnormal deliveries (prolonged for more than 24 hours, and/or maternal distress with remaining pups in uterus, which sometimes caused maternal death in labor). "Abnormal (died)" means maternal death by PPD3, and "Abnormal (euthanized)" means maternal health deterioration necessitating euthanasia by PPD3.

D: The scores for nest quality, indicated by the use of nest material and nest shape (see Methods) at PPD 0.

E: The placentophagia score (1, all the live pups were cleaned for amniotic membrane, umbilical cord, and placenta; 0.5, partially; 0, none of the pups were cleaned) at PPD 0.

F: The spontaneous pup grouping score (1, all the live pups were grouped in the nest; 0.5 , the live pups were in the nest except one; 0 , all the live pups were outside of the nest.) at PPD 0.

G: The pup survival rate (number of live pups at PPD 1 divided by the number of live pups at PPD 0 morning) of each mother.

H: Schematic of the experimental timeline and responses to pup exposure in mothers. Only four of them $\left(\mathrm{Oxt}^{+/+}-\operatorname{Trh}^{+/-}: 1, O x t^{-/-}-\operatorname{Trh}^{+/-}: 2, O x t^{-{ }_{-}} \operatorname{Trh}^{-/-}: 1\right)$ had expereinced the pup-exposure assay as a virgin, whose results are included in Fig. 3A.

I: Responses to pup exposure in virgin males.

$\mathbf{J}$ : Responses to pup exposure in males, which were separated from their female mates immediately after copulation. Copulation was assesed by vaginal plugs in females. Only males whose mates were later confirmed as pregnant are shown.

K: Fertility ratios of the males after mating (7 days of cohabitation with females).

L: Responses to pup exposure in males, which were separated from their female mates after 7 days of cohabitation.

For D-H, data from only mothers who had normal delivery are shown. For J-L, data from only males who exhibited infanticide as a virgin are shown.

$(+/ *)$ means wild-type $(+/+)$ or heterozygous $\mathrm{KO}(+/-)$, and (-/-) means homozygous $\mathrm{KO}$ for the designated genetic locus. A black vertical bar in schematic of the experimental timelines indicates the day a pup-exposure assay was performed. Error bars represent the mean \pm SEM. Different letters denote significant differences (multiple comparisons by Fisher's exact test for categorical data, and Welch's $t$-test for continuous data), $p<0.05$. Numbers of animals used are described in the figure. 
941

942

943

944

945

946

947

948

949

950

951

952

953

954

955

956

957

958

959

960

961

962

963

964

965

966

967

968

969

970

971

972

973

974

Figure 4. Pup-directed behaviors of the virgin and postpartum Oxt-Trh-Avpr1a-Avpr1b quadruple KO (QKO) female mice in the standard pup-exposure assay.

A: Schematic of the experimental timeline. "Post-isolation (PI)": the mothers tested 13 days after the isolation from pups at PPD1. A black vertical bar indicates the day a pup-exposure assay was performed.

B: Gross pup-directed behaviors in each genotype, with the number of mice for each behavioral category in each genotype.

C-G: Details of pup-directed behaviors compared between quadruple (-/-)) vs. all other genotypes (others) (C); heterozygous (+/-) versus homozygous KO (-/-) for Avprlb (D), Avprla (E), Trh (F), and $O x t(\mathbf{G})$, regardless of other genetic loci. The left three variables on the abscissae are latencies, the right three variables are proportions of the time bins in which the indicated behaviors were performed.

Error bars represent the mean \pm SEM. Fisher's exact test for categorical data; Welch's $t$-test for continuous data, $* * p<0.01, * p<0.05$. Numbers of animals used are described in the figure.

Figure 5. Pup-directed behaviors of the virgin and postpartum Oxtr-Avprla-Avprlb triple KO (TKO) female mice.

A: Schematic of the experimental timeline. "Post-isolation (PI)": the mothers tested 13 days after the isolation from pups at PPD1. A black vertical bar indicates the day a pup-exposure assay was performed.

B: Gross pup-directed behaviors in each genotype, with the number of mice for each behavioral category in each genotype.

C-G: Details of pup-directed behaviors compared between triple (-/-)) vs. all other genotypes (others) (C); triple (-/-) vs $\mathrm{Oxtr}^{+/-} ; \operatorname{Avprla}^{+/-} ; \operatorname{Avprlb}^{+/-}$(triple (+/-)) (D), Avprlb (E), Avprla (F), and Oxtr (G), regardless of other genetic loci. The left three variables on the abscissae are latencies, the right three variables are proportions of the time bins in which the indicated behaviors were performed.Error bars represent the mean \pm SEM. Fisher's exact test for categorical data; Welch's $t$-test for continuous data, ${ }^{* *} p<0.001,{ }^{* *} p<0.01, * p<0.05$. Numbers of animals used are described in the figure. 
Figure 6. Oxt (neurophysin I, NPI) and AVP expressions in the MPOA were not affected by Oxtr or Avpr genetic targeting.

Distribution of NPI and AVP (black)-ir cells in the MPOA of Avprla-Oxtr-Avprlb triple mutant (TKO) female mice. triple (+/-) mean Avprla ${ }^{+/-} ;$Oxtr $^{+/-} ; A v p r 1 b^{+/-}$, triple (-/-) mean Avprla ${ }^{-/-}$; Oxtr $^{-/-}$; Avprlb $b^{-/-}$TKO virgin female mice. (A) The sections were stained by IHC. AC (B, E), SO (C, F) and PVH (D, G was identified by counterstain using NeuN. All panels are arranged in anteriorposterior order. (B-G) High-magnification images of same-colored squares. (H-K) The numbers of NPI-ir neurons in the AC $(\mathbf{H})$, SO $(\mathbf{I})$ and PVH $(\mathbf{J})$, and of Avp-ir neurons in the PVH (K) of triple $(+/-)(\mathrm{N}=4)$ and $(-/-)(\mathrm{N}=4)$ virgin females. Error bars represent the mean $\pm \mathrm{SEM}$.

Figure 7. Responses to pups in the standard pup-exposure assay with the virgin and copulated Oxtr-Avpr1a-Avpr1b triple KO (TKO) male mice.

A: Schematics of the experimental timeline. A black vertical bar indicates the day a pup-exposure assay was performed.

B-F: Gross pup-directed behaviors in each genotype, with the number of mice for each behavioral category in each genotype. Oxtr ${ }^{-/-}$; Avprla ${ }^{-/-}$; Avprlb ${ }^{-/-}$(triple (-/-)) vs all other genotypes (others) (B); triple (-/-) vs $\mathrm{Oxtr}^{+/-}$; Avprla ${ }^{+/-} ; \operatorname{Avprlb}^{+/-}$(triple $\left.\left(^{+/-}\right)\right)(\mathbf{C})$; heterozygous (+/-) vs. homozygous (-/-) KO for each four genetic loci for Avprlb (D), Avprla $(\mathbf{E})$, and Oxtr $(\mathbf{F})$, regardless of other genetic loci.

Blue asterisk: Fisher's exact test between parental (pups retrieved) vs. non-parental (infanticide or no pup retrieved); red asterisk: Fisher's exact test between infanticidal vs. non-infanticidal; ${ }^{*} p<0.05$.

\section{Figure 8. Confirmation of the results with HIR strain of Oxtr-Avpr1a-Avpr1b triple KO mice.}

A, B: Responses to pups, schematics of the experimental timeline, latencies, and proportion of time of pup-directed behaviors in the standard pup-exposure assay in virgin (A) and postpartum (B) females. The left three variables are latencies, the right three variables are proportion of time bins performed the indicated behavior.

C, D: Responses to pups and schematic of the experimental timeline of pup-directed behaviors in the standard pup-exposure assay in virgin males (C) and father mice (D).

A black vertical bar in schematic of the experimental timelines indicates the day a pup-exposure assay was performed. Error bars represent the mean \pm SEM. Fisher's exact test for categorical data; Welch's $t$-test for continuous data, $* * p<0.01,{ }^{*} p<0.05$. Numbers of animals used are described in 
1009

1010

1011

1012

1013

1014

1015

1016

1017

1018

1019

1020

1021

1022

1023

1024

1025

1026

1027

1028

1029

1030

1031

1032

1033

1034

1035

1036

1037

the figure.

Figure 9. Effect of restraint stress on the pup-directed behaviors of the virgin and postpartum Oxtr-Avpr1a-Avpr1b triple KO (TKO) female mice.

A: Gross pup-directed behaviors in each genotype, with the number of mice for each behavioral category in each genotype.

B-D: Details of pup-directed behaviors compared between heterozygous (+/-) versus homozygous KO (-/-) for Avprlb (B), Avprla (C), and for Oxtr (D), disregarding other genetic loci. The left two variables are latencies, the right two variables are proportion of time bins performed the indicated behavior.

E: Total amount of pup retrieval behavior, measured by the total time the 3 pups spent in the nest. Different letters denote significant differences (multiple comparisons by Welch's $t$-test, $p<0.05$ ). See also the Extended Data Figure 9-1 for the results of Kruskal-Wallis rank sum tests with post hoc multiple comparisons by Nemenyi test.

Error bars represent the mean \pm SEM. Welch's $t$-test for continuous data; blue asterisk: Fisher's exact test between parental (pups retrieved) versus non-parental (infanticide or no pup retrieved), ${ }^{*} p<0.05$. Different letters denote significant differences (multiple comparisons by Welch's $t$-test for continuous data), $p<0.05$. Numbers of animals used are described in the figure.

\section{Figure 10. Stress sensitivity of the virgin Oxtr-Avprla-Avpr1b triple KO (TKO) female and male} mice.

Behavioral characteristics in the open-field test $(\mathbf{A}, \mathbf{D})$ and elevated plus maize $(\mathbf{B}, \mathbf{E})$, and effect of restraint stress on plasma corticosterone level $(\mathbf{C}, \mathbf{F})$. (+/-) means heterozygous, (-/-) means homozygous, and * means (+/-) or (-/-) KO for the designated genetic locus.

Error bars represent the mean \pm SEM. Welch's $t$-test, $* p<0.05$. Numbers of animals used are described in the figure. 
1038 Table 1. Primers used for genotyping.

1039

\begin{tabular}{|c|c|c|c|}
\hline strain & gene loci & primer sequence ( $5^{\prime}$ to $\left.3^{\prime}\right)$ & $\mathrm{Tm}$ \\
\hline \multirow{3}{*}{ B6;129S-Oxt $t^{t m I W s y} / \mathrm{J}$} & \multicolumn{3}{|c|}{ gtgctggacctggatatgcgcaag } \\
\hline & Oxt & agcgtcctttgccgcccgggccgcaggggagacactgtggctgtgg & 68 \\
\hline & & ctgctaaagcgcatgctccagactgc & \\
\hline \multirow{3}{*}{$\operatorname{Trh} \mathrm{KO}$} & \multirow{3}{*}{$\operatorname{Trh}$} & tctcgtcgtgacccatggcgatg & \multirow{3}{*}{64} \\
\hline & & tctcgtcgtgacccatggcgatg & \\
\hline & & ttactcctccagaggttccctgac & \\
\hline \multirow{3}{*}{ B6.129P2-Avprla ${ }^{\text {tmIDgen }} / \mathrm{J}$} & \multirow{3}{*}{ Avpr la } & cgcaacgaggagctggcgaagctgg & \multirow{3}{*}{64} \\
\hline & & gcggtaggtgatgtcccagcacagc & \\
\hline & & gggccagctcattcctcccactcat & \\
\hline \multirow{3}{*}{ Oxtr KO } & \multirow{3}{*}{ Oxtr } & gttgggaacagcggtgatta & \multirow{3}{*}{64} \\
\hline & & ccttggaagcaggaggtgaag & \\
\hline & & gctgcgcagtggtggtgacttc & \\
\hline \multirow{3}{*}{ B6;129X1-Avprlb ${ }^{t m l W s y} / \mathrm{J}$} & \multirow{3}{*}{ Avpr $1 b$} & accccttcccagcctctgagcccagaaagcgaagg & \multirow{3}{*}{64} \\
\hline & & gaaacggctactctctccgattccaaaagaaag & \\
\hline & & acctgtagatatttgacagcccgg & \\
\hline \multirow{6}{*}{$\mathrm{B} 6 ; 129 \mathrm{~Sv}-V l a^{\text {tmlGzt}} V 1 b^{t m l G z t}$} & \multirow{3}{*}{ Avpr $1 a$} & acaagtgttttgtaactagtgactcta & \multirow{3}{*}{60} \\
\hline & & aggggettctggtcacgccttgt & \\
\hline & & acatagcgttggctacccgtgat & \\
\hline & \multirow{3}{*}{ Avpr $1 b$} & gaaacggctactctctcgattccaaaagaag & \multirow{3}{*}{60} \\
\hline & & gcgaattcgatatcaagcttatcga & \\
\hline & & acctgtagatatttgacagcccgg & \\
\hline
\end{tabular}

1040 
1041 1042

Table 2. Mouse genetic mutant lines and control genotypes used in each Figure.

\begin{tabular}{|c|c|c|c|c|c|}
\hline Figure & Abbreviation & KO mice & Source & Genotype of control groups & Comment \\
\hline \multirow[t]{2}{*}{ Fig. 3} & DKO & $\operatorname{Trh} \mathrm{KO}$ mice & Yamada et al. & $\operatorname{Trh}^{+/- \text {or }-/} ; O x t^{+/- \text {or }-l}$ & $+/$ means $^{+/+}$or $^{+/-}$ \\
\hline & & Oxt $\mathrm{KO}$ mice & JAX (\#2713) & & \\
\hline \multirow[t]{5}{*}{ Fig. 4} & QKO & Avpr la KO mice & JAX (\#5776) & (Fig. 4C) All other genotypes besides Avprla ${ }^{-1-}$ AvprIb ${ }^{-1} ; \mathrm{Trh}^{-1} ; \mathrm{Oxt}^{-1-}$ & \\
\hline & & Avpr $1 b$ KO mice & JAX (\#6160) & (Fig. 4D) Avprla $a^{+/- \text {or }-/} ; \mathrm{AvprIb}^{+/-} ; \mathrm{Trh}^{+/- \text {or }-/-} ; \mathrm{Oxt}^{+/- \text {or }-/-}$ & \\
\hline & & $\operatorname{Trh} \mathrm{KO}$ mice & Yamada et al. & (Fig. 4E) Avprla $a^{+/ /} ; \mathrm{Avprlb}^{+/- \text {or }-/ /} ; \mathrm{Tr}^{+/ \text {or }-/} ; O x t^{+/-o r-/-}$ & \\
\hline & & Oxt KO mice & JAX (\#2713) & (Fig. 4F) Avprla $^{+/- \text {or }-/} ; \operatorname{Avprlb}^{+/- \text {or }-/} ; \operatorname{Trh}^{+/} ; O x t^{+/- \text {or } / /}$ & \\
\hline & & & & 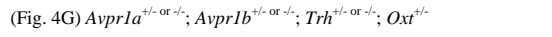 & \\
\hline \multirow[t]{5}{*}{ Fig. 5} & TKO & Avpr la KO mice & JAX (\#5776) & (Fig. 5C) All other genotypes besides Avprla ${ }^{-1-}$; pvrl $^{-1-} ;$ Oxtr $^{-1-}$ & \\
\hline & & Avpr $1 b$ KO mice & JAX (\#6160) & (Fig. 5D) $\mathrm{Avprla}^{+/} ; \mathrm{Avprlb}^{+/-} ; \mathrm{Oxtr}^{+/-}$ & \\
\hline & & Oxtr KO mice & Takayanagi et al. & (Fig. 5E) Avprla ${ }^{+/- \text {or }-/} ; \mathrm{Avprlb}^{+/ /} ; \mathrm{Oxtr}^{+/ \text {or }-/}$ & \\
\hline & & & & (Fig. 5F) Avprla ${ }^{+/-}$Avprlb ${ }^{+/- \text {or }-/} ; \mathrm{Oxtr}^{+/- \text {or }-/}$ & \\
\hline & & & & (Fig. 5G) Avprla ${ }^{+/ \text {or- }-/} ; \mathrm{AvprIb}^{+/ / \text {or }-/} ; \mathrm{Oxtr}^{+/-}$ & \\
\hline \multirow[t]{3}{*}{ Fig. 6} & TKO & Avpr la KO mice & JAX (\#5776) & Avprla $^{+/-} ;$Avprlb $^{+/-} ;$Oxtr $^{+/-}$ & \\
\hline & & Avpr $1 b$ KO mice & JAX (\#6160) & & \\
\hline & & Oxtr KO mice & Takayanagi et al. & & \\
\hline \multirow[t]{5}{*}{ Fig. 7} & TKO & Avpr $1 a$ KO mice & JAX (\#5776) & (Fig. 7B) All other genotypes besides Avprla ${ }^{-1} ; A v p r I b^{--} ; O x r^{-1-}$ & \\
\hline & & Avpr $1 b$ KO mice & JAX (\#6160) & (Fig. 7C) Avprla $a^{+/} ;$Avprlb $^{+/-} ; \mathrm{Oxtr}^{+/-}$ & \\
\hline & & Oxtr KO mice & Takayanagi et al. & (Fig. 7D) Avprla ${ }^{+/- \text {or }-/ /} ; \mathrm{Avprlb}^{+/ /} ; \mathrm{Oxtr}^{+/ / \text {or }-/}$ & \\
\hline & & & & (Fig. 7E) Avprla ${ }^{+/-} ; \mathrm{Avprlb}^{+/- \text {or }-/ /} ; \mathrm{Oxtr}^{+/ / \text {or }-/}$ & \\
\hline & & & & (Fig. 7F) Avprla $a^{+/- \text {or }-/ /} ;$ Avprlb $^{+/- \text {or }-/-} ;$ Oxtr $^{+/-}$ & \\
\hline
\end{tabular}




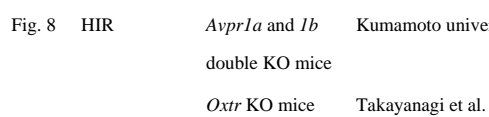

JAX (\#5776) Avprla KO line was not a null mutation, Kumamoto (\#599) Avprla KO line has been confirmed for the phenotype in cardiovascular system.

\begin{tabular}{|c|c|c|c|c|}
\hline \multirow[t]{3}{*}{ Fig. 9} & TKO & Avpr la KO mice & JAX (\#5776) & (Fig. 9B) Avprla ${ }^{+/- \text {or }-/-} ; \mathrm{Avprlb}^{+/-} ; \mathrm{Oxtr}^{+/- \text {or }-/-}$ \\
\hline & & Avpr $1 b$ KO mice & JAX (\#6160) & (Fig. 9C) Avprla ${ }^{+/-} ;$Avprlb $^{+/- \text {or }-/-} ; \mathrm{Oxtr}^{+/- \text {or }-/}$ \\
\hline & & Oxtr KO mice & Takayanagi et al. & (Fig. 9D) Avprla ${ }^{+/- \text {or-l-l}} ; \mathrm{Avprlb}^{+/- \text {or }-/ /} ; \mathrm{Oxtr}^{+/-}$ \\
\hline \multirow[t]{4}{*}{ Fig. 10} & TKO & Avpr la KO mice & JAX (\#5776) & (Large panels) $\mathrm{Avprla}^{++} ; \mathrm{AvprIb}^{+/-} ; \mathrm{Oxtr}^{+/-}$ \\
\hline & & Avpr $1 b$ KO mice & JAX (\#6160) & (Small left panels) Avprla $a^{+/- \text {or }-/} ;$ Avprlb $^{+/- \text {or }-/} ; \mathrm{Oxtr}^{+/-}$ \\
\hline & & Oxtr KO mice & Takayanagi et al. & (Small middle panels) Avprla ${ }^{+/-} ;$Avprll $^{+/- \text {or }-/ /} ; \mathrm{Oxtr}^{+/- \text {or }-/-}$ \\
\hline & & & & (Small right panels) Avprlat ${ }^{+/ \text {or }-/} ; \operatorname{Avprlb}^{+/-} ; O x t r^{+/ \text {or }-/}$ \\
\hline
\end{tabular}

1043

1044 
1045 Figure 9-1. Effects of restraint stress on the pup-directed behaviors of the virgin 1046 Oxtr-Avpr1a-Avpr1b (TKO) triple mutant female mice with non-parametric statistical analysis.

Parental behavior, measured by the total time the 3 pups spent in nest, of 1048 Oxtr-Avprla-Avprlb triple mutant virgin female mice in standard condition (a) and after restraint 1049 stress (b). $(+/-)$ means heterozygous, (-/-) means homozygous, and * means (+/-) or (-/-) for the designated 1051 genetic locus. ${ }^{*} \mathrm{HH}$ means $\left(\right.$ Avprla ${ }^{*}, \mathrm{Oxtr}^{+/}, \mathrm{Avprlb}^{+/}$; gray with white stripe), ${ }^{*} \mathrm{KH}$ means (Avprla ${ }^{*}$, $\mathrm{Oxtr}^{-/-}$, Avprlb $^{+/-}$; black with white stripe), *HK means (Avprla ${ }^{*}, \mathrm{Oxtr}^{+/-}$, Avprlb $^{-/-}$; gray), and *KK means (Avprla ${ }^{*}$, Oxtr $^{-/}$, Avprlb ${ }^{-/-}$; black).

1054

Results of non-parametric Kruskal-Wallis rank sum tests are shown above the graphs. \# and \#\#

1055 denote significant differences between two groups in post hoc multiple comparisons (Nemenyi test; \# $p<0.05, \# \# p<0.01)$.

Nemenyi tests were performed by the R package PMCMR ver. 4.3 (Pohlert, 2014). The box plots were generated by geom_boxplot function with default settings in R package ggplot2 ver. 3.1.0 (Wickham, 2016). The medians are represented by the horizontal bar in the middle of each box. The lower and upper hinges correspond to the first and third quartiles (the 25th and 75th percentiles). The 1061 upper whisker extends from the hinge to the largest value no further than $1.5 *$ IQR from the hinge. 1062 The lower whisker extends from the hinge to the smallest value at most $1.5 *$ IQR of the hinge. Data 1063 beyond the end of the whiskers are called "outlying" points and are plotted individually (Wickham, 2016). 

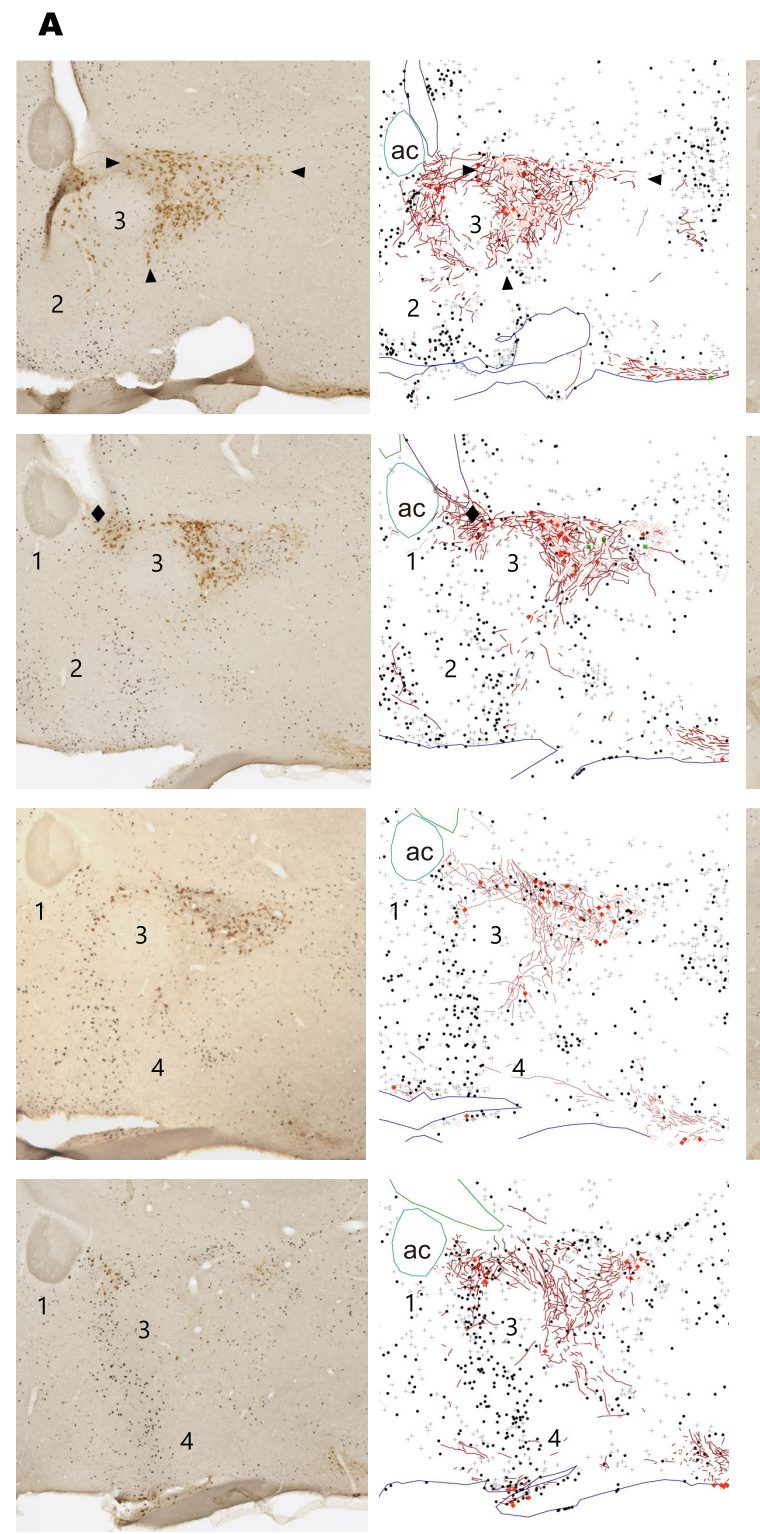

(2)
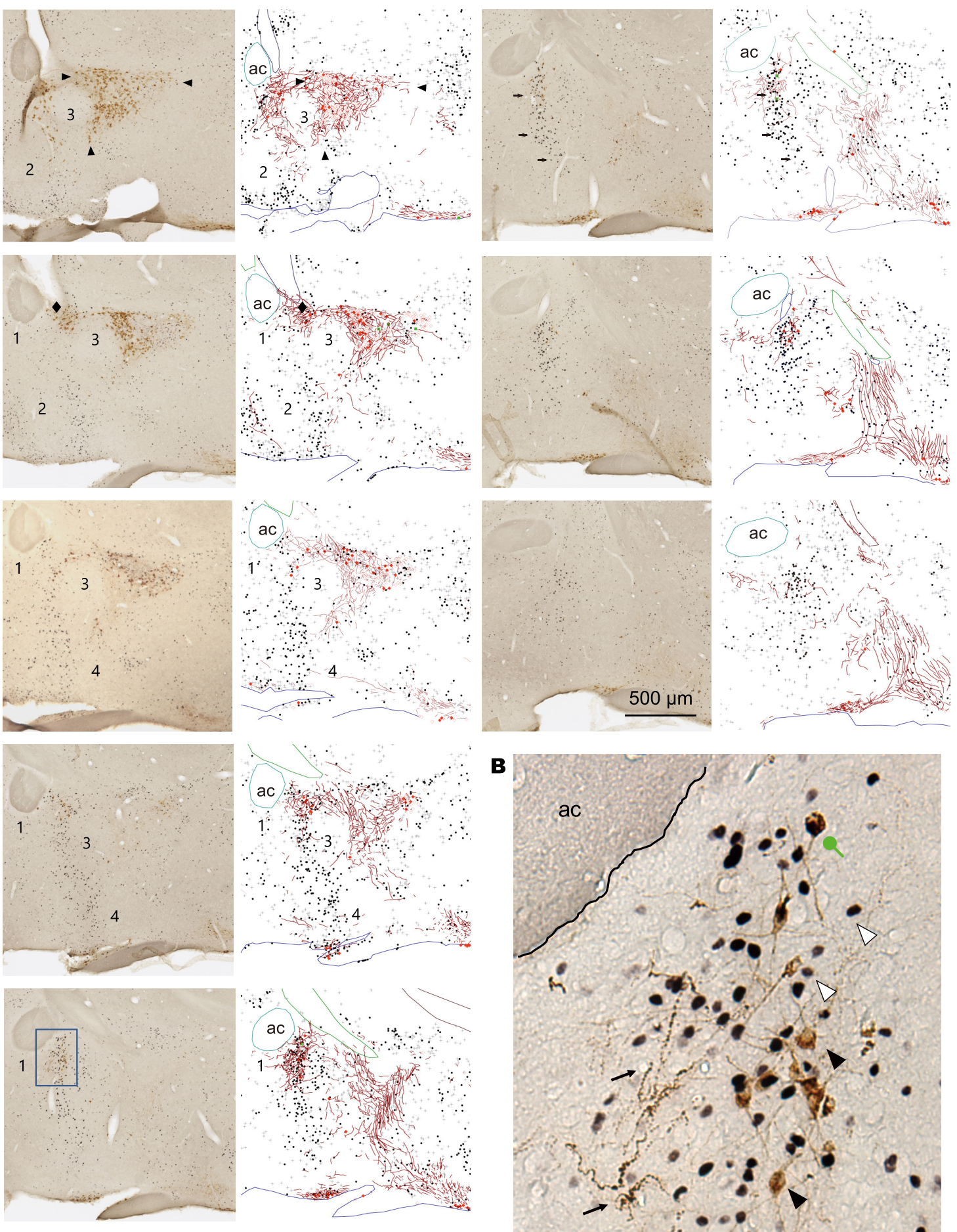

U

\section{B}

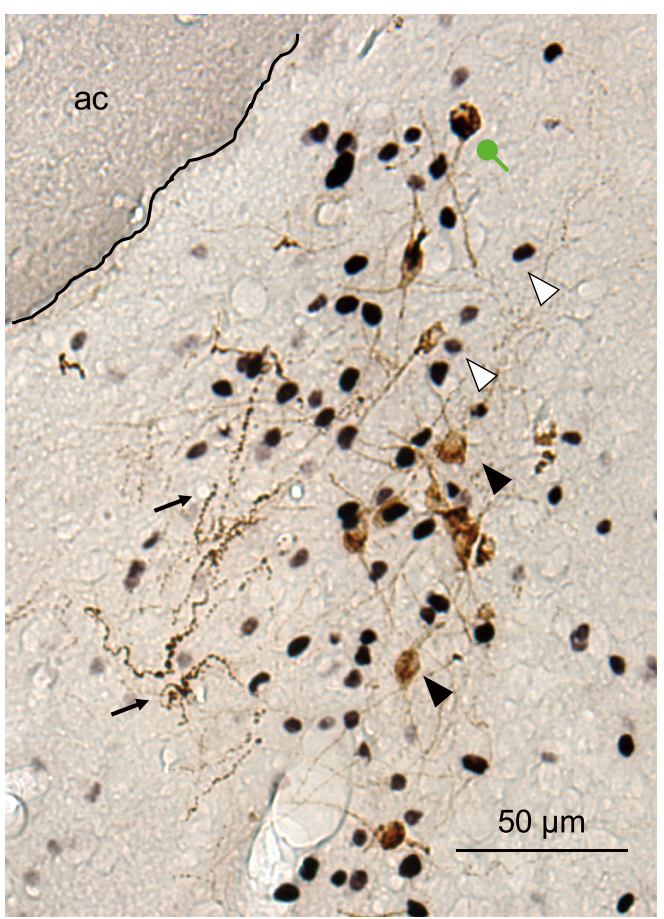



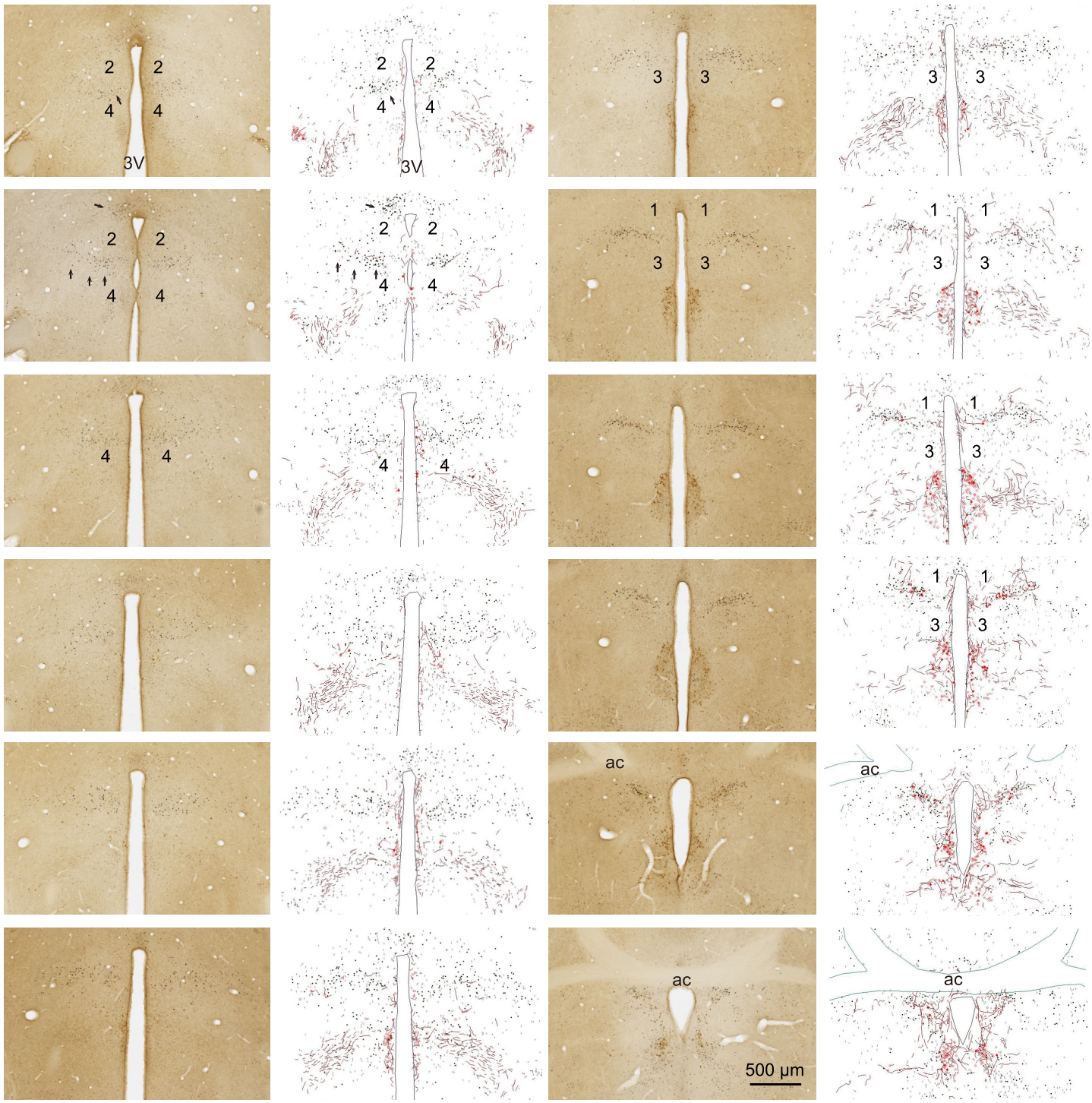

(

극

(a)

(1) 


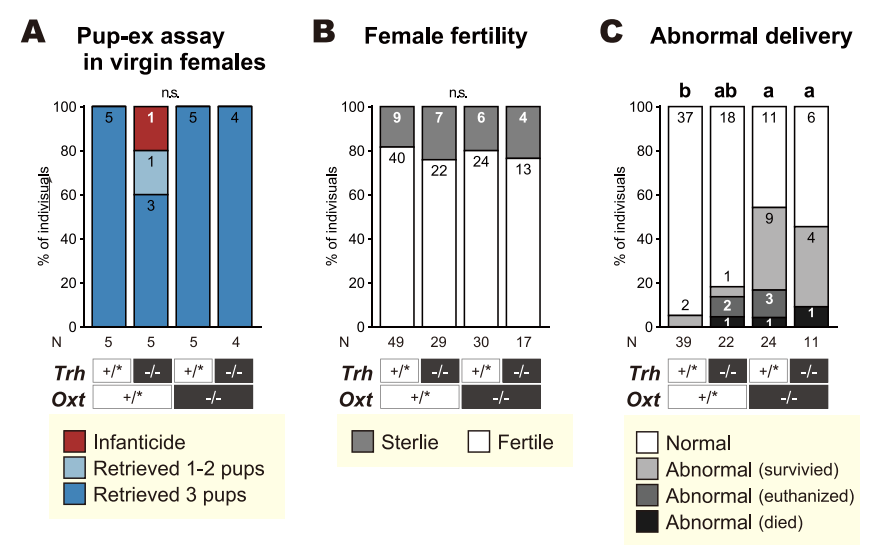

H Pup-ex assay in mothers at PPD0-1
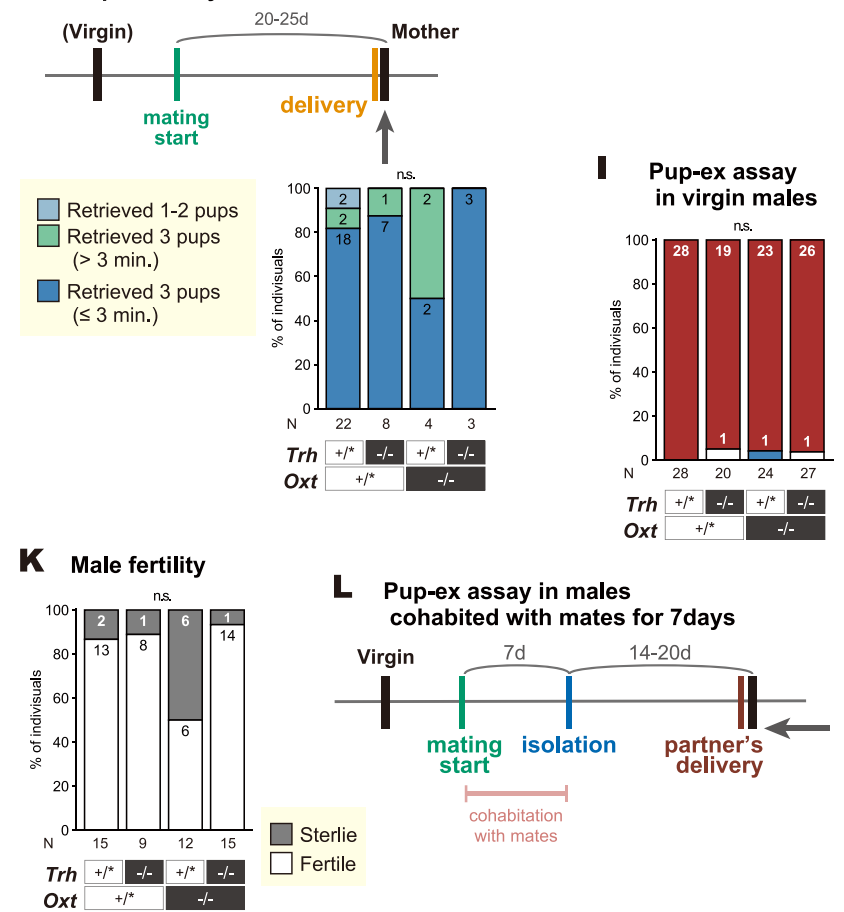

$L$ Pup-ex assay in males
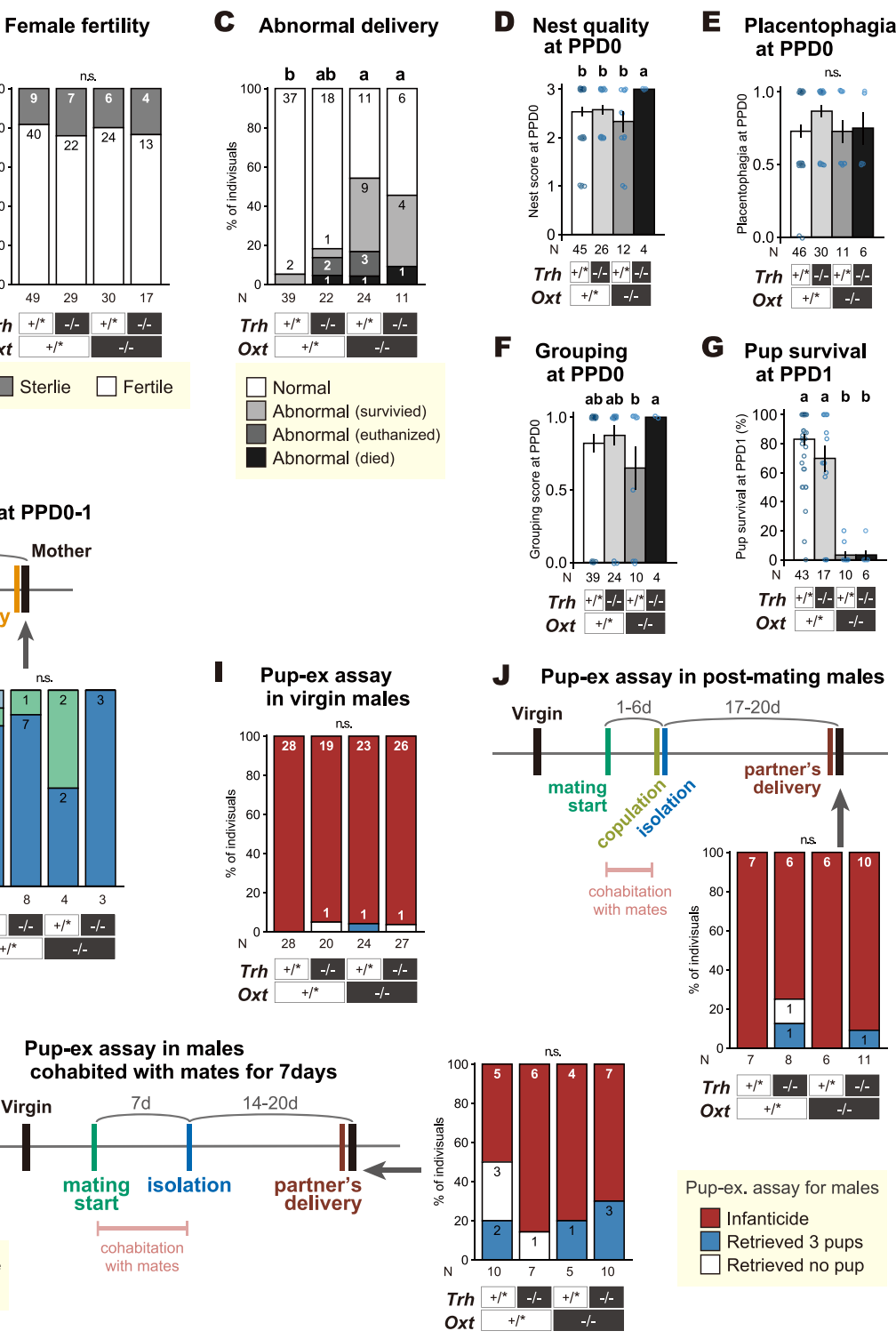

Grouping
at PPD0

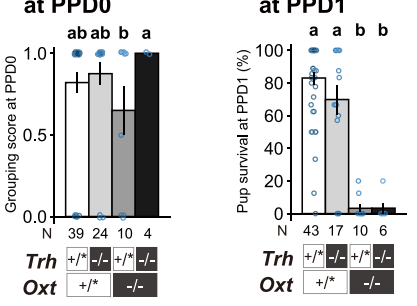

J Pup-ex assay in post-mating males
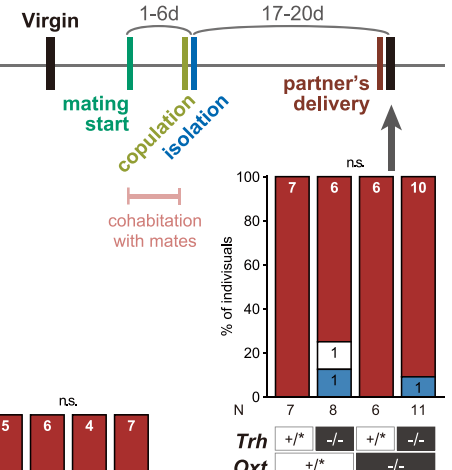

Pup-ex. assay for males

$\square$ Infanticide

Retrieved 3 pups

$\square$ Retrieved no pup 

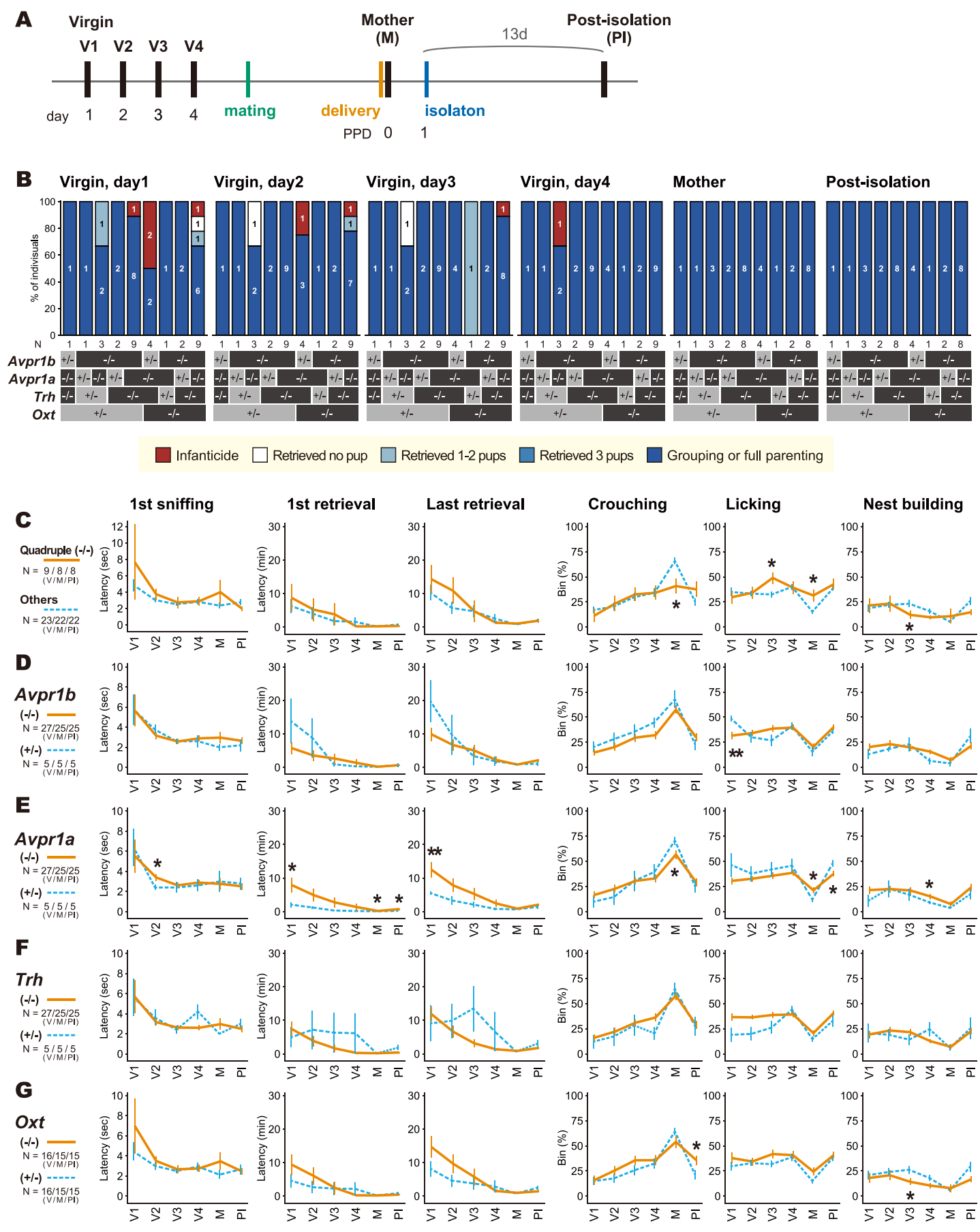

$\mathbf{V}=$ virgin, $\mathbf{M}=$ mother, $\mathbf{P I}=$ post-isolation 

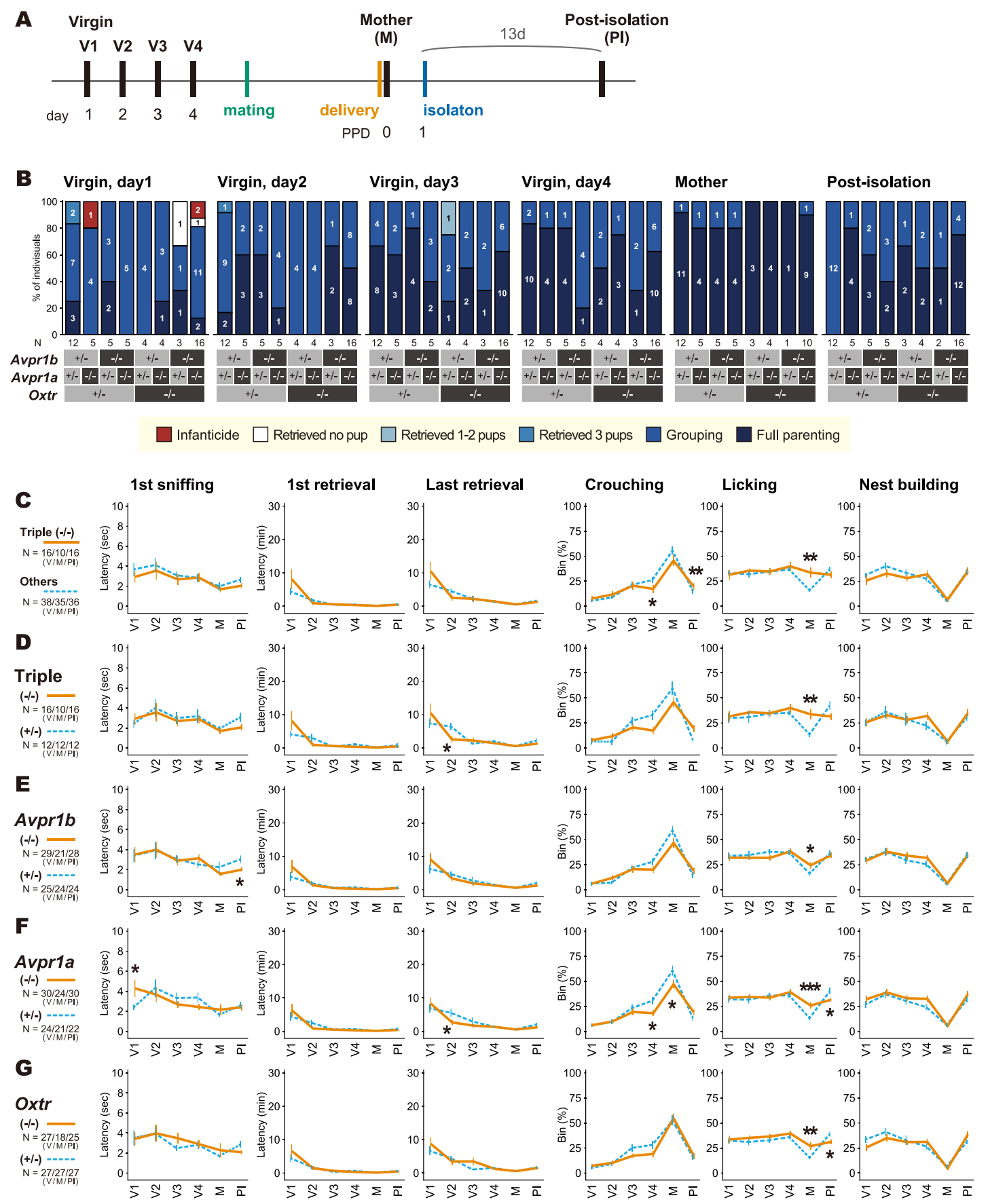

$\mathbf{V}=$ virgin, $\mathbf{M}=$ mother, $\mathbf{P I}=$ post-isolation 
A
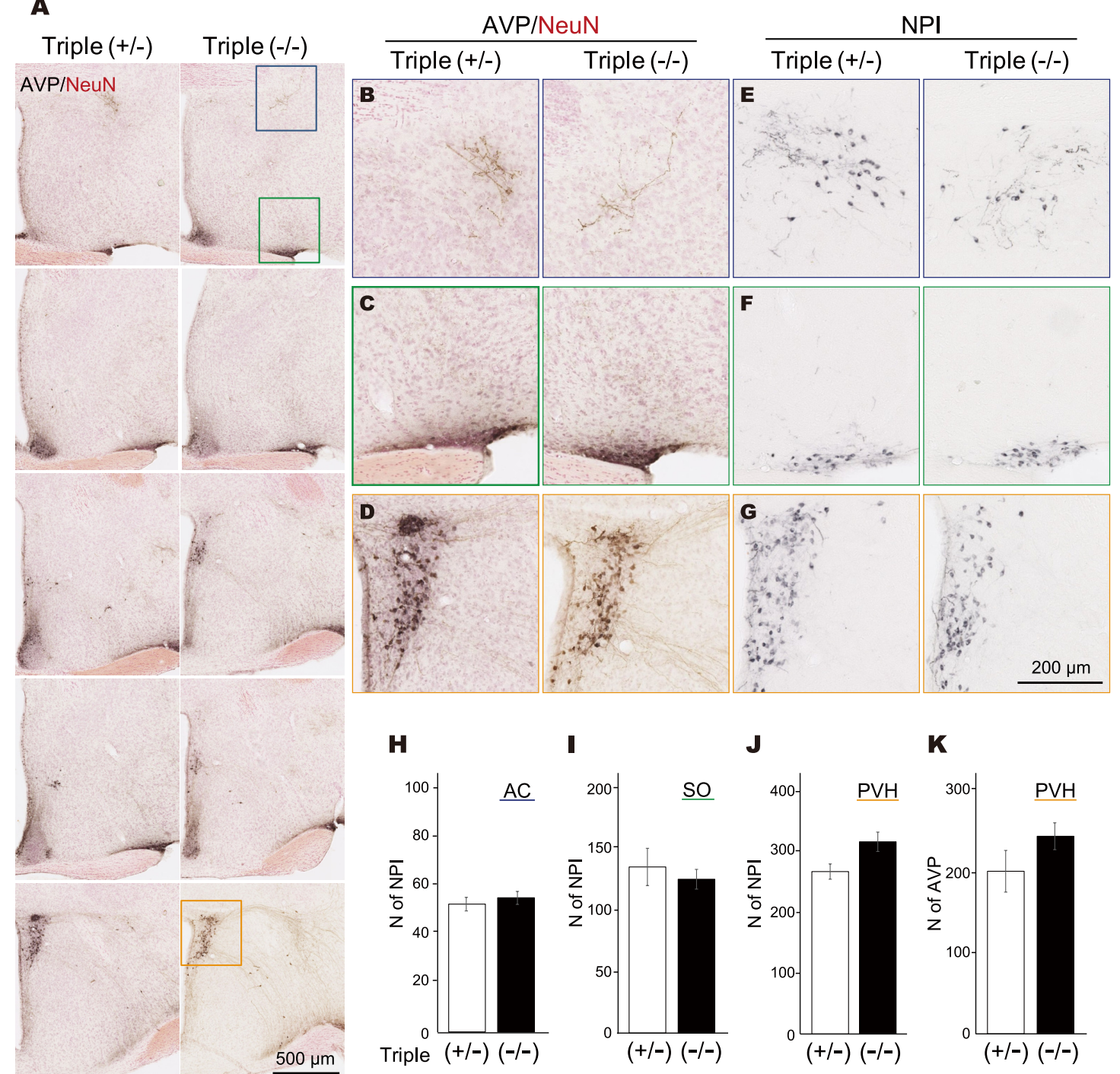

I

J

K
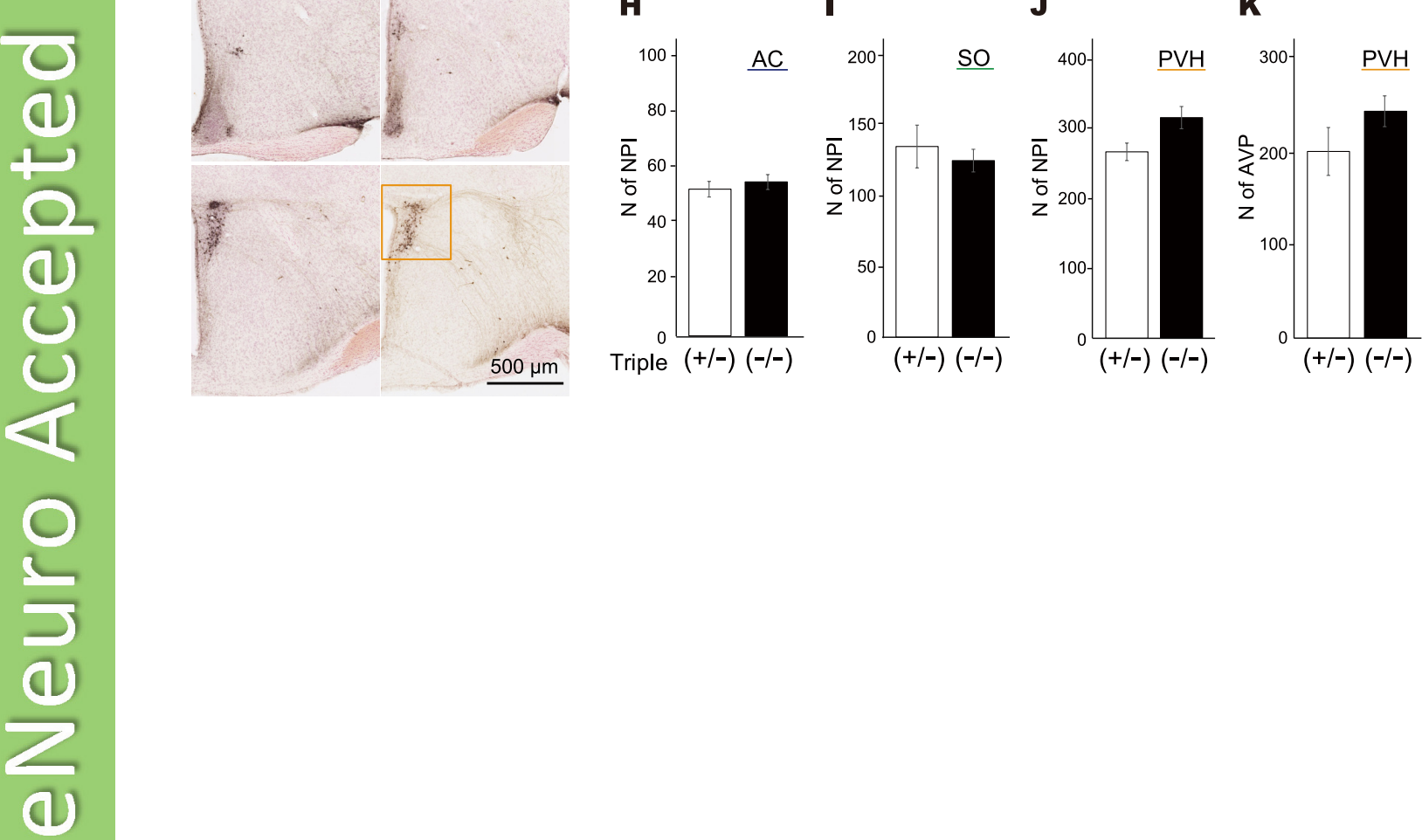

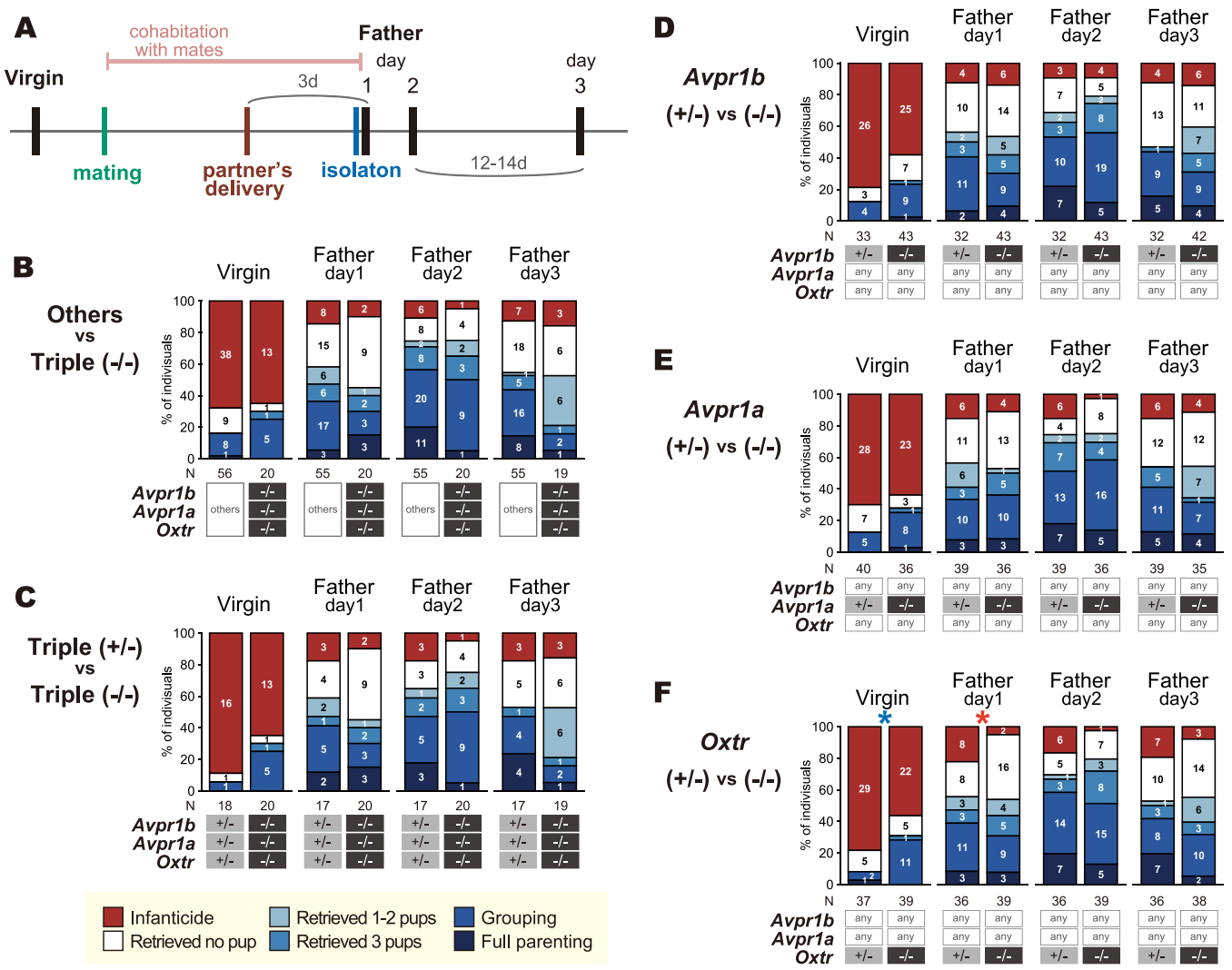
Triple (-/-)

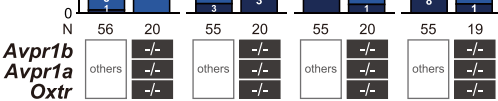

Retrieved no pup $\square$ Retrieved 3 pups $\quad$ Full parenting 
A Virgin female: triple (+/-) vs (-/-)

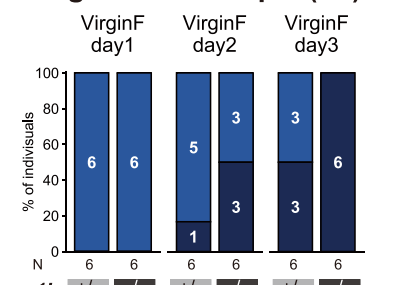

$\square$ Grouping

Full parenting

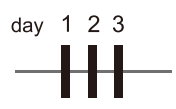

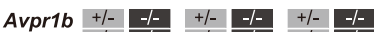

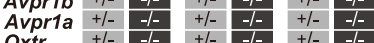

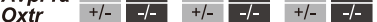
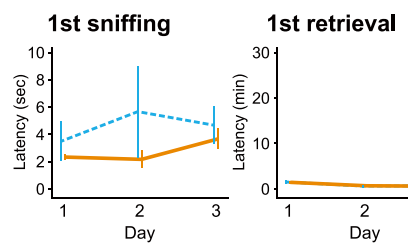

C Virgin male: triple (+/-) vs (-/-)

$\begin{array}{ccc}\begin{array}{c}\text { VirginM } \\ \text { day1 }\end{array} & \begin{array}{c}\text { VirginM } \\ \text { day2 }\end{array} & \begin{array}{c}\text { VirginM } \\ \text { day3 }\end{array}\end{array}$

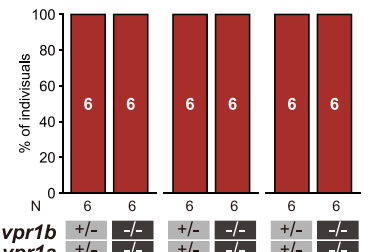

$\square$ Infanticide

day $\begin{array}{lll}1 & 2 & 3\end{array}$
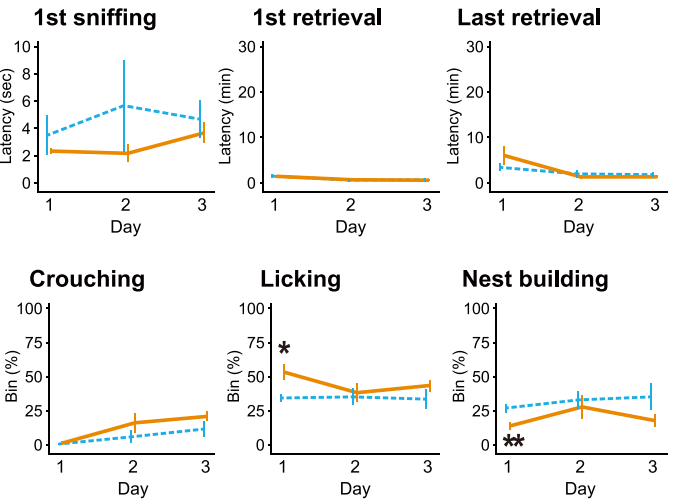

Nest building

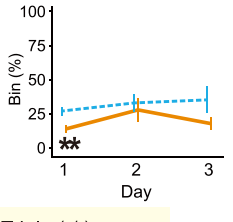

11
B Mother: triple (+/-) vs (-/-)

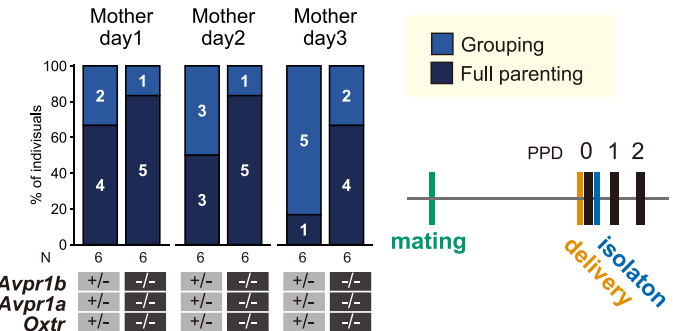

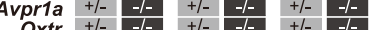
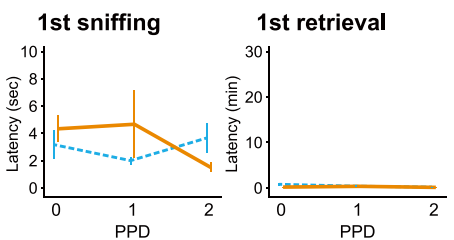

Last retrieval
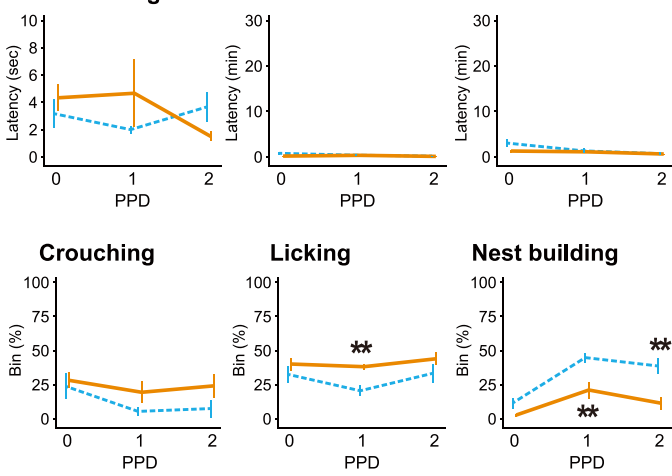

Nest building

100

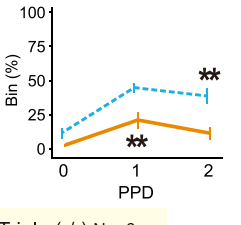

-.-.-. Triple (+/-) $\mathrm{N}=6 \quad$ Triple $(-/-) \mathrm{N}=6$

D Father: triple (+/-) vs (-/-)

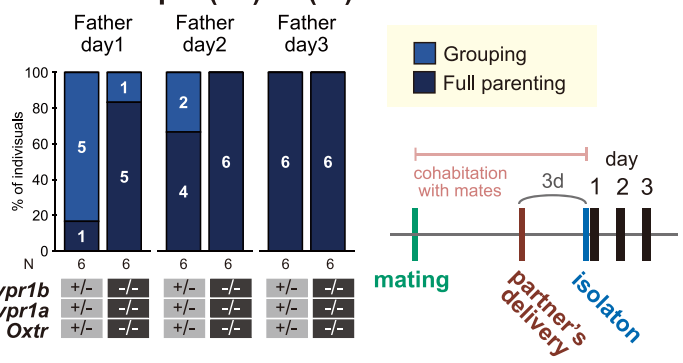




\section{A Pup-ex assay after restraint stress}
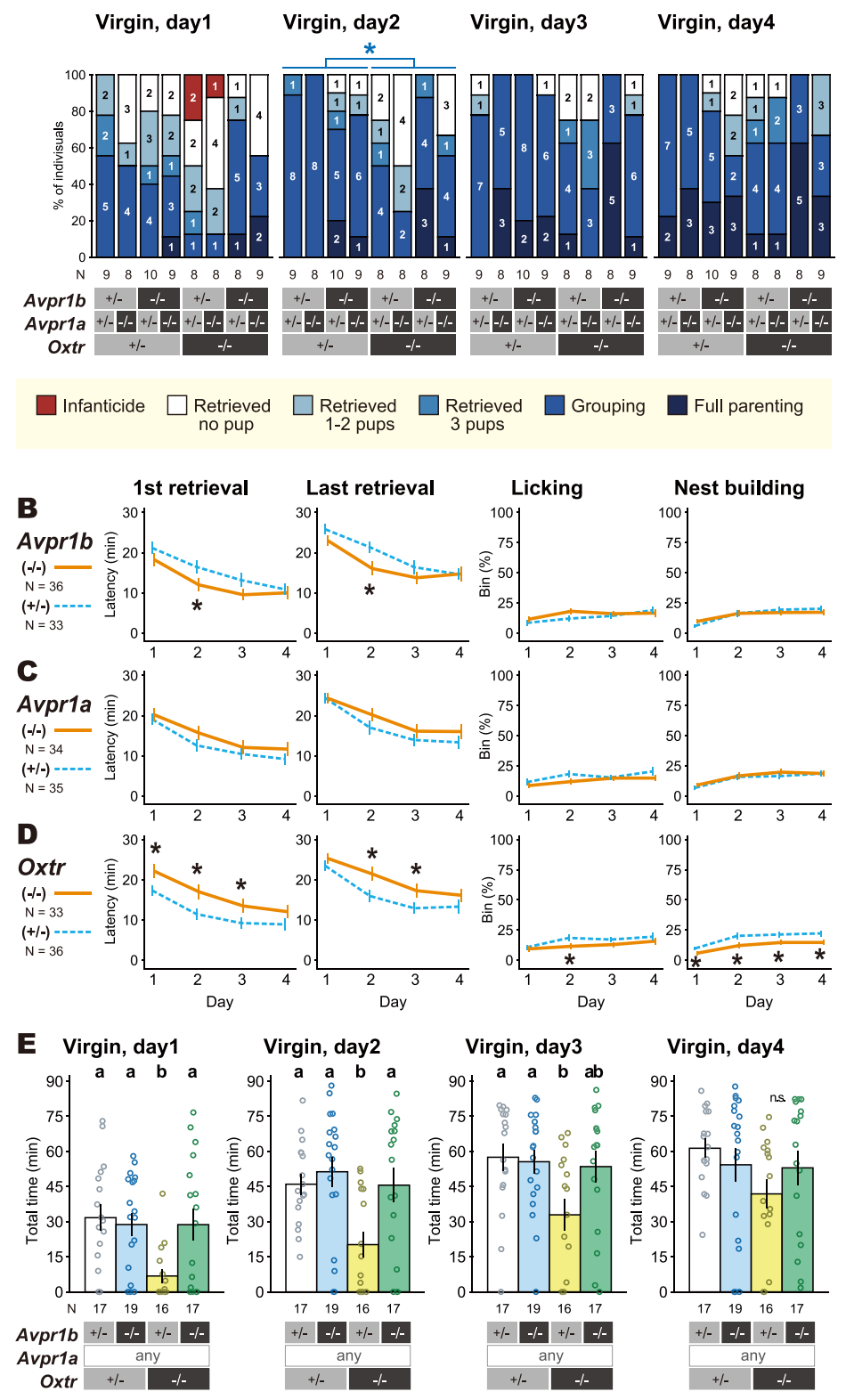

Virgin, day4

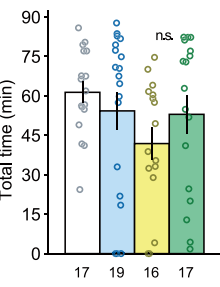

$+/-\mid-1-x+/-17-1-$ 


\section{A Female: Open Field Test} Total distance

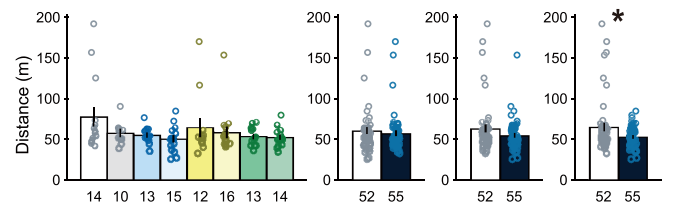

Time in center

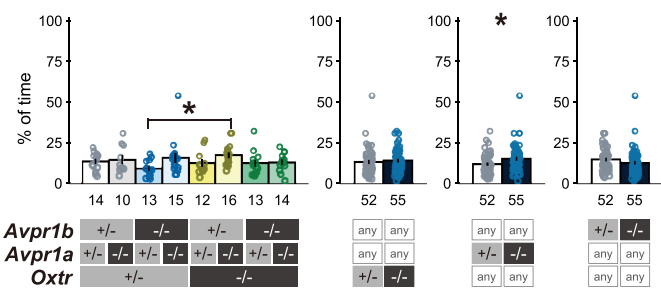

B Female: Elevated Plus Maze Total distance

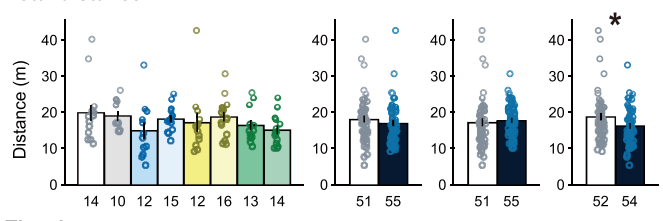

Time in open arms

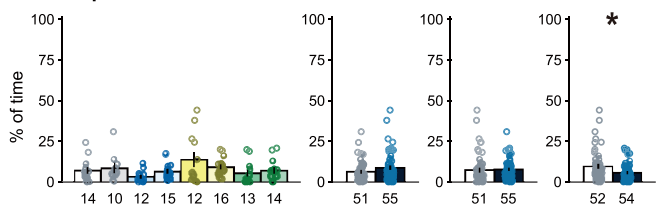

Entries to open arms

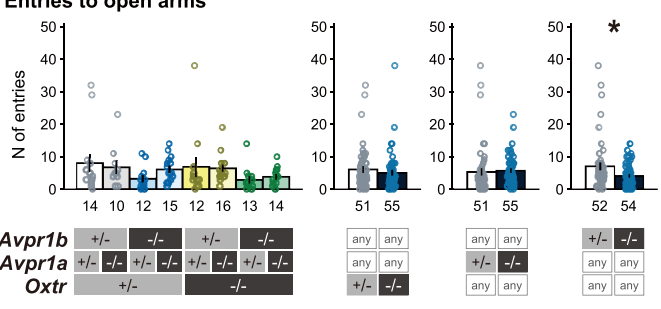

C Female: plasma cortisol level Condition: no stress

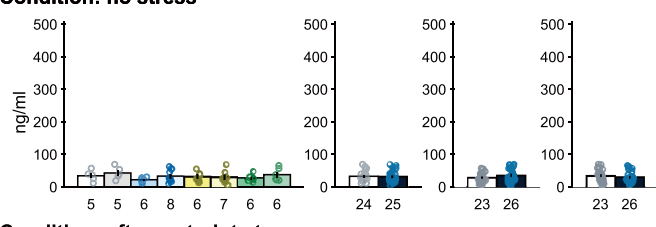

Condition: after restraint stress
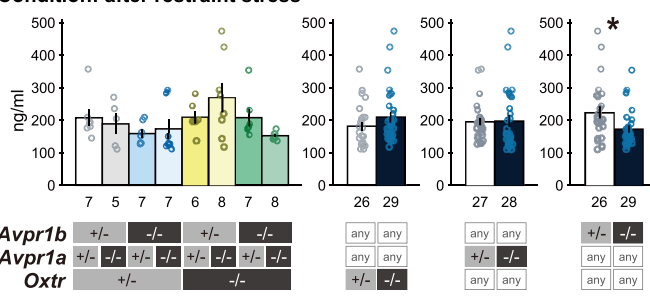

D Male: Open Field Test

Total distance

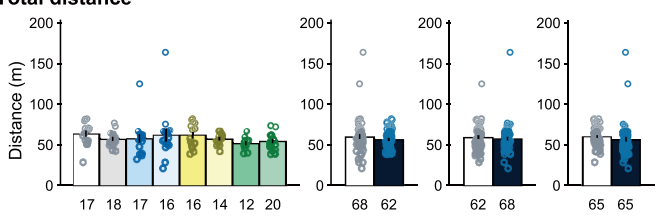

Time in center

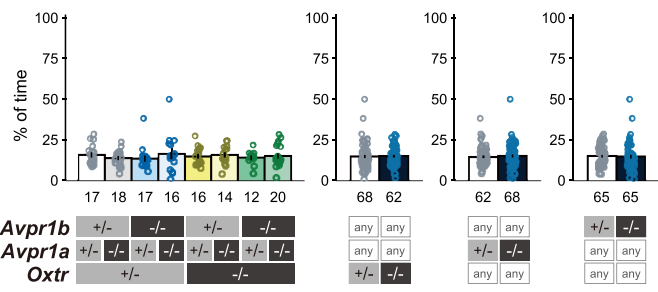

E Male: Elevated Plus Maze

Total distance

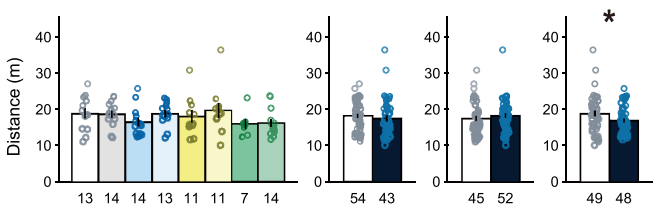

Time in open arms

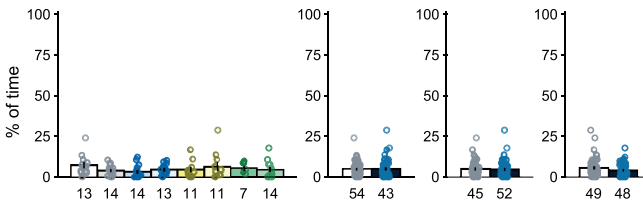

Entries to open arms

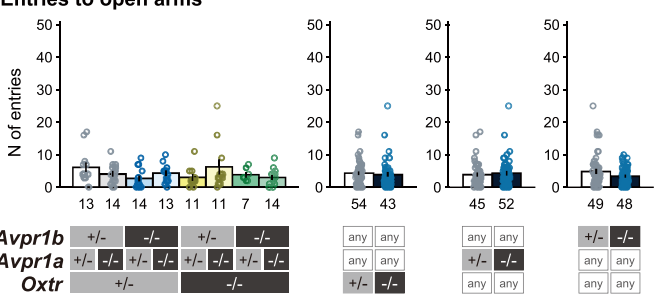

F Male: plasma cortisol level

Condition: no stress

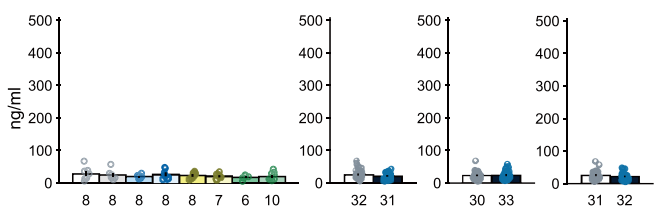

Condition: after restraint stress

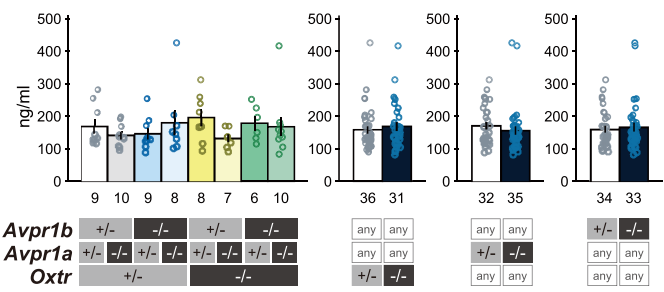




\begin{tabular}{|c|c|c|c|}
\hline strain & gene loci & primer sequence (5' to $3^{\prime}$ ) & $\mathrm{Tm}$ \\
\hline \multirow[b]{2}{*}{ B6;129S-Oxt ${ }^{t m l W s y} / \mathrm{J}$} & & gtgctggacctggatatgcgcaag & \\
\hline & $O x t$ & $\begin{array}{c}\text { agcgtcctttgccgcccgggccgcaggggagacactgtggctgtgg } \\
\text { ctgctaaagcocatgctccagactgc }\end{array}$ & 68 \\
\hline \multirow{3}{*}{$\operatorname{Trh} \mathrm{KO}$} & & tctcgtcgtgacccatggcgatg & \\
\hline & Trh & tctcgtcgtgacccatggcgatg & 64 \\
\hline & & ttactcctccagaggttccctgac & \\
\hline \multirow{3}{*}{ B6.129P2-Avprla ${ }^{\text {tmlDgen }} / \mathrm{J}$} & & cgcaacgaggagctggcgaagctgg & \\
\hline & Avpr $1 a$ & gcggtaggtgatgtcccagcacagc & 64 \\
\hline & & gggccagctcattcctcccactcat & \\
\hline \multirow{3}{*}{ Oxtr KO } & & gttgggaacagcggtgatta & \\
\hline & Oxtr & ccttggaagcaggaggtgaag & 64 \\
\hline & & gctgcgcagtggtggtgacttc & \\
\hline \multirow{3}{*}{$\mathrm{B} 6 ; 129 \mathrm{X} 1-A v p r l b^{t m l W s y} / \mathrm{J}$} & & accccttcccagcctctgagcccagaaagcgaagg & \\
\hline & $A v p r 1 b$ & gaaacggctactctctccgattccaaaagaaag & 64 \\
\hline & & acctgtagatatttgacagcccgg & \\
\hline \multirow{6}{*}{ B6;129Sv-Vla $a^{t m I G z t} V l b^{t m I G z t}$} & & acaagtgttttgtaactagtgactcta & \\
\hline & Avpr la & aggggcttctggtcacgccttgt & 60 \\
\hline & & acatagcgttggctacccgtgat & \\
\hline & & gaacggctactctctcgattccaaagaag & \\
\hline & Avpr $1 b$ & gcgaattcgatatcaagcttatcga & 60 \\
\hline & & acctgtagatatttgacagcccgg & \\
\hline
\end{tabular}


Table 2. Mouse genetic mutant lines and control genotypes used in each Figure.

\begin{tabular}{|c|c|c|c|c|c|}
\hline Figure & Abbreviation & KO mice & Source & Genotype of control groups & Comment \\
\hline \multirow[t]{2}{*}{ Fig. 3} & DKO & Trh KO mice & Yamada et al. & $\operatorname{Trh}^{+/- \text {or }-/-} ; O x t^{+/- \text {or }-/-}$ & ${ }^{+/ *}$ means $^{+/+}$or $^{+/-}$ \\
\hline & & Oxt $\mathrm{KO}$ mice & JAX (\#2713) & & \\
\hline \multirow[t]{5}{*}{ Fig. 4} & QKO & Avpr $1 a$ KO mice & JAX (\#5776) & (Fig. 4C) All other genotypes besides AvprIa $a^{--} ; A v p r I b^{--} ; \operatorname{Trh}^{-/} ; O x t^{--}$ & \\
\hline & & Avpr $1 b$ KO mice & JAX (\#6160) & (Fig. 4D) Avprla ${ }^{+/- \text {or }-/} ; \mathrm{Avprlb}^{+/-} ; \mathrm{Trh}^{+/- \text {or }-/} ; \mathrm{Oxt}^{+/ / \text {or }-/}$ & \\
\hline & & $\operatorname{Trh} \mathrm{KO}$ mice & Yamada et al. & (Fig. 4E) $\mathrm{Avprla}^{+/ /} ; \mathrm{Avprll}^{+/- \text {or }-/} ; \mathrm{Trh}^{+/- \text {or }-/} ; \mathrm{Oxt}^{+/-o r-/}$ & \\
\hline & & Oxt KO mice & JAX (\#2713) & (Fig. 4F) Avprla ${ }^{+/ \text {or }-/-} ; A v p r I b^{+/- \text {or }-/} ; \operatorname{Trh}^{+/ /} ; O x t^{t /- \text { or }-/}$ & \\
\hline & & & & (Fig. 4G) Avprla $a^{+/- \text {or }-/} ; \mathrm{AvprIb}^{+/- \text {or }-/} ; \mathrm{Trh}^{+/ \text {or }-/} ; \mathrm{Oxt}^{+/-}$ & \\
\hline \multirow[t]{5}{*}{ Fig. 5} & TKO & Avpr la KO mice & JAX (\#5776) & (Fig. 5C) All other genotypes besides Avprla $a^{-/} ; A v p r l b^{--} ; O_{x t r^{-1}}$ & \\
\hline & & Avpr $1 b$ KO mice & $\mathrm{JAX}(\# 6160)$ & (Fig. 5D) Avprla ${ }^{+/} ;$Avprlb $^{+/-} ;$Oxtr $^{+/-}$ & \\
\hline & & Oxtr KO mice & Takayanagi et al. & (Fig. 5E) Avprla $a^{+/- \text {or }-/} ; \mathrm{Avprlb}^{+/-} ; \mathrm{Oxtr}^{+/- \text {or }-/-}$ & \\
\hline & & & & (Fig. 5F) Avprla ${ }^{+/-} ; \mathrm{Avprlb}^{+/- \text {or }-/ /} ; \mathrm{Oxtr}^{+/- \text {or }-/}$ & \\
\hline & & & & (Fig. 5G) Avprla ${ }^{+/- \text {or }-/} ;$ Avprl $^{+/- \text {or }-/} ; \mathrm{Oxtr}^{+/-}$ & \\
\hline \multirow[t]{3}{*}{ Fig. 6} & TKO & Avpr la KO mice & JAX (\#5776) & Avprla $^{+/} ;$AvprIb $^{+/-} ;$Oxtr $^{+/-}$ & \\
\hline & & Avpr $1 b$ KO mice & JAX (\#6160) & & \\
\hline & & Oxtr KO mice & Takayanagi et al. & & \\
\hline \multirow[t]{5}{*}{ Fig. 7} & TKO & Avpr la $\mathrm{KO}$ mice & JAX (\#5776) & 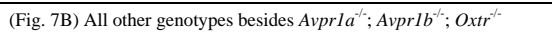 & \\
\hline & & Avpr $1 b$ KO mice & JAX (\#6160) & (Fig. 7C) Avprla ${ }^{+/-} ;$Avprlb $^{+/-} ;$Oxtr $^{+/-}$ & \\
\hline & & Oxtr KO mice & Takayanagi et al. & (Fig. 7D) Avprla ${ }^{+/- \text {or }-/ /} ; \mathrm{Avprlb}^{+/-} ; \mathrm{Oxtr}^{+/-\mathrm{or}-/}$ & \\
\hline & & & & (Fig. 7E) Avprla ${ }^{+/-} ; \mathrm{Avprlb}^{+/- \text {or }-/ /} ; \mathrm{Oxtr}^{+/- \text {or }-/}$ & \\
\hline & & & & (Fig. 7F) Avprla $a^{+/- \text {or }-/ /} ;$ Avprlb $^{+/- \text {or }-/-} ;$ Oxtr $^{+/-}$ & \\
\hline \multirow[t]{3}{*}{ Fig. 8} & HIR & Avprla and $l b$ & Kumamoto university (\#559) & Avprla $^{+/-} ;$Avprlb $^{+/} ;$Oxtr $^{+/-}$ & JAX (\#5776) Avprla KO line was not a null mutation, \\
\hline & & double KO mice & & & Kumamoto (\#599) AvprIa KO line has been confirmed \\
\hline & & Oxtr KO mice & Takayanagi et al. & & for the phenotype in cardiovascular system. \\
\hline \multirow[t]{3}{*}{ Fig. 9} & TKO & Avpr la KO mice & JAX (\#5776) & (Fig. 9B) Avprla ${ }^{+/- \text {or }-/} ;$ Avprll $^{+/} ; \mathrm{Oxtr}^{+/ \text {or }-/-}$ & \\
\hline & & Avpr $1 b$ KO mice & $\mathrm{JAX}(\# 6160)$ & (Fig. 9C) Avprla ${ }^{+/-} ; \mathrm{Avprlb}^{+/- \text {or }-/} ; \mathrm{Oxtr}^{+/- \text {or }-/}$ & \\
\hline & & Oxtr KO mice & Takayanagi et al. & (Fig. 9D) Avprla $a^{+/- \text {or }-/ /} ; \mathrm{Avprlb}^{+/- \text {or }-/} ; \mathrm{Oxtr}^{+/-}$ & \\
\hline Fig. 10 & TKO & Avpr la KO mice & JAX (\#5776) & (Large panels) Avprla $^{+/} ;$AvprIb $^{+/-} ;$Oxtr $^{+/-}$ & \\
\hline
\end{tabular}




\begin{tabular}{|c|c|c|}
\hline Avpr $1 b$ KO mice & JAX (\#6160) & (Small left panels) $A v p r l a^{+/- \text {or }-l} ; A v p r l b^{+/ \text {or }-l /} ; O x t r^{+/-}$ \\
\hline Oxtr $\mathrm{KO}$ mice & Takayanagi et al. & 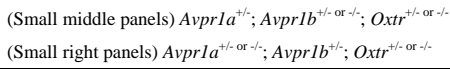 \\
\hline
\end{tabular}

